\title{
Modelo de optimización LFSR basado en método de coeficientes adaptativos para sistemas reconfigurables de ERNC
}

\author{
LFSR optimization model based on the adaptive coefficients \\ method for ERNC reconfigurable systems
}

\author{
Cecilia Sandoval-Ruiz ${ }^{1 *}$
}

Recibido 18 de enero de 2021, aceptado 6 de septiembre de 2021

Received: January 18, 2021 Accepted: September 6, 2021

\begin{abstract}
RESUMEN
En esta investigación se presenta un modelo de optimización para sistemas de energías renovables, considerando la configuración híbrida de conversión, etapas de almacenamiento y esquema de realimentación, basada en coeficientes adaptativos de la arquitectura LFSR (Linear Feedback Shift Register). El método de correlación entre los módulos de conversión, para tecnología fotovoltaica bifacial y eólica, comprende el modelado neuronal y un análisis de las estructuras componentes, para establecer las ecuaciones descriptivas. Entre los resultados se reporta la caracterización de los módulos para un esquema concatenado, simplificación del nivel de abstracción y correspondencia entre los modelos del sistema híbrido realimentado. Entre los aportes se presenta una descripción matemática generalizada, con operadores de convolución LFC $(n, k)$ y una propuesta de optimización, a partir de eficiencia energética. Se obtiene como conclusión que el modelo reconfigurable con realimentación selectiva, cumple con los criterios de recuperación de energía regenerativa y reutilización de componentes, para la adaptación de las tecnologías a modelos de desarrollo sostenible.
\end{abstract}

Palabras clave: Modelo de optimización, esquemas reconfigurables LFSR, coeficientes adaptativos, energías renovables, fotovoltaica bifacial.

\section{ABSTRACT}

In this research an optimization model for renewable energy systems is presented, considering the hybrid configuration of conversion, storage stages and feedback scheme, based on adaptive coefficients of the LFSR (Linear Feedback Shift Register) architecture. The correlation method between the conversion modules, for bifacial and wind photovoltaic technology, comprises the neural model and an analysis of the component structures, to establish the descriptive equations. The results include the characterization of the modules for a concatenated scheme, simplification of the level of abstraction and correspondence between the models of the hybrid feedback system. Among the contributions is a generalized mathematical description, with $\operatorname{LFC}(\mathrm{n}, \mathrm{k})$ convolution operators and an optimization proposal, based on energy efficiency. The conclusion is that the reconfigurable model with selective feedback meets the criteria for regenerative energy recovery and reusable components, for the adaptation of technologies to sustainable development models.

Keywords: Optimization model, reconfigurable LFSR schemes, adaptive coefficients, renewable energies, bifacial photovoltaic bPV.

\footnotetext{
1 Facultad de Ingeniería. Universidad de Carabobo. Venezuela. E-mail: cesandova@gmail.com

* Autor de correspondencia: cesandova@gmail.com
} 


\section{INTRODUCCIÓN}

Investigaciones recientes están orientadas al desarrollo de modelos matemáticos para el soporte de la optimización y configuración dinámica de los arreglos fotovoltaicos [1-2]. Del mismo modo, se han estudiado alternativas de migración de la matriz energética convencional a sistemas híbridos de energías y su potencial para reconversión a tecnologías de energías renovables no convencionales - ERNC [3]. Por tal motivo, se considera que una descripción matemática de la composición estructural de estos sistemas, basada en coeficientes adaptativos, permitirá definir un esquema reconfigurable, el cual soportará actualizaciones y optimizaciones dinámicas.

Al momento de describir los sistemas de ERNC, se ha reconocido patrones similares en la arquitectura de los módulos, que corresponden a operadores matemáticos con estructura LFSR (Linear Feedback Shift Register), tanto a nivel del sistema, como de componentes. De esta manera, para la caracterización de los convertidores de energía se podrá generalizar la relación de señales que interactúan entre los módulos concatenados, a fin de definir una matriz de coeficientes para las etapas paralelas de operadores funcionales, así como la configuración de registros de almacenamiento y realimentación, para el manejo de bloques discretos de energía.

Para alcanzar un modelo válido para el comportamiento de cada etapa del sistema de ERNC, se definen parámetros adaptativos, que pueden ser identificados como los coeficientes físicos del modelo teórico y pueden ser estimados, a partir de ensayos prácticos, siendo éste uno de los aspectos que contempla la presente investigación. La innovación del modelo viene dada por la comparación entre subsistemas del sistema de ERNC, lo que ha permitido identificar estructuras auto-similares, de forma específica, en arreglos de fotovoltaica bifacial y arreglos de convertidores eólicos, que permiten definirlos como sistemas realimentados, siendo que esta interpretación de una turbina como un arreglo inteligente de registro desplazamiento de realimentación lineal no ha sido previamente establecida en el área de investigación.

Se presenta un factor diferenciador con trabajos previos [1-2], basado en ensayo práctico que permite la estimación de parámetros de los sistemas de fotovoltaica bifacial y eólico, aplicando redes neuronales - ANN. De esta forma, se ha podido definir un método para la obtención de los coeficientes, dentro del arreglo de optimización. Estos resultados pueden ser extrapolados a otros sistemas, a través del modelo para la integración de técnica de optimización y nuevas tecnologías, a partir del reconocimiento de la arquitectura común entre los esquemas.

Existe un compromiso entre el modelo teórico con parámetros técnicos y el modelo práctico, con variables dadas por las condiciones de las instalaciones, este es el motivo por el que se diseñan parques científicos. En esta oportunidad se propone una etapa teórica con herramientas software y etapa de modelado experimental y optimización dinámica, sobre hardware, que combina ambos conceptos de forma eficiente. Los objetivos planteados en la investigación corresponden a establecer una relación directa entre los sistemas fotovoltaicos - SFV, sistemas de turbinas eólica y sistemas de almacenamiento de ERNC, a través de un esquema realimentado de múltiples etapas, analizando las coincidencias estructurales entre las diversas tecnologías de conversión de ERNC, así definir un modelo para los componentes del sistema de potencia, ensayar un método de modelado neuronal, bajo un enfoque modular de la ANN para simplificar el nivel de abstracción y proponer la optimización de los sistemas de ERNC, basado en coeficientes adaptativos y realimentación selectiva LFSR.

\section{ANTECEDENTES EN MODELADO NEURONAL DE SISTEMAS DE ERNC}

Actuales investigaciones se orientan al modelado neuronal en aplicaciones fotovoltaicas [4-7], destacando variaciones de redes convolucionales LSMT (Long-Short Term Memory) [8] y otras configuraciones de redes recurrentes. Las RNN están basadas en el modelo de Hopfield, el cual está definido a partir de una analogía eléctrica con el comportamiento neuronal, donde cada neurona tiene su salida realimentada, a través de un elemento de retardo, hacia otras neuronas de la red y presenta un término dinámico de capacitancia en la salida, que representa la memoria de la neurona. En estas alternativas de modelado neuronal se puede observar un alto nivel de abstracción en las redes de aprendizaje profundo. Para esta investigación 
se busca disminuir el nivel de abstracción, a fin de proponer una optimización en base a los fundamentos teóricos de las etapas del sistema.

Algunos trabajos [9] desarrollan modelado neuronal para mejorar el rendimiento de producción de energía, tal es el caso de la optimización basado en modelos $\mathrm{PV} / \mathrm{T}$ de enfriamiento que utilizan nano fluidos, a partir del análisis de impacto de la irradiancia solar y temperatura ambiente. Así mismo, se aplican arquitecturas de redes profundas, las cuales generan modelos composicionales, donde el patrón a reconocer, es expresado como una composición de niveles, lo que se ha considerado como punto de partida de esta investigación. Para la obtención del modelo de optimización, se considera el modelado, de forma semi-empírica, cuyos coeficientes pueden ser obtenidos por datos experimentales, en relación a las principales variables o parámetros con mayor impacto [10], un análisis de correspondencia a través de un método de generalización e identificación con las características físicas.

Para ello se ha definido una estructura LFSR multinivel como arquitectura del modelo, la cual aporta características estructurales similares a las redes convolucionales, con un tratamiento modular de las etapas (conocido el conjunto de datos). Esto permite reconocer la correspondencia entre las capas del modelo y las etapas del sistema realimentados de conversión y almacenamiento de ERNC, para establecer la matriz de pesos sinápticos en relación a los coeficientes físicos de los componentes del sistema, lo que facilita la identificación de los parámetros a optimizar en el modelo del sistema.

\section{MODELO DEL ARREGLO FOTOVOLTAICO BIFACIAL}

En [11] presentan una revisión de la fotovoltaica bifacial - bPV, resaltando la importancia de modelado numérico de esta tecnología como soporte para simulación y optimización de diseño, con descripción de las capas componentes del módulo bifaciales, en el que destaca la diferencia de bPERC (bifacial Passivated Emitter Rear Cell), que permite recuperar los fotones no convertidos. Por su parte, en [12] se desarrolló un modelo óptico de radiación directa, difusa y reflejada, eléctrico, de cinco parámetros, y térmico, redes de transferencia de calor, de los módulos fotovoltaicos bifaciales, con énfasis en las múltiples variables físicas. En [13] se presenta el estudio detallado de los factores y distribución de los paneles en el arreglo FV [14-15], que influyen en la eficiencia de conversión, por lo que la reconfiguración del sistema y cada una de las técnicas de optimización pueden ser relacionadas con coeficientes del modelo, para su adaptación dinámica. Estos avances evidencian la necesidad de optimización basada en modelo, para establecer el polinomio descriptor, se inicia por una interpretación de los componentes, en este punto, la tecnología bifacial como sistema realimentado LFSR (Ver Tabla 1) y las variables de optimización [1] para el modelado neuronal.

De esta manera la configuración de las cadenas del arreglo se ajustaría a la condición óptima, desacoplando los módulos que presenten fallas o sombreado, a través de la matriz de conmutación del arreglo - MCA con control basado en operadores neuronales LFC [16-18]. A nivel de tecnología en [1] se propone optimización por coeficientes de temperatura en relación a parámetros eléctricos $\mathrm{TC}_{\mathrm{ISC}}$ y $\mathrm{TC}_{\mathrm{VOC}}$, donde se plantean coeficientes de recuperación de calor para el panel fotovoltaico, así como la aplicación de técnicas de sensibilización espectral [2]. En la etapa de salida del convertidor la optimización se realiza con técnicas de seguimiento del punto de máxima potencia - MPPT [1], por arreglo o por cada panel FV.

De esta manera, se identifican aplicaciones de control para la optimización del sistema fotovoltaico, en cada una de sus etapas. Finalmente, para el modelo planteado se tiene el estudio del Coeficiente de Bifacialidad [19], la relación existencial que hay entre el $I_{s c}, V_{o c}$ y la $P_{\text {máx }}$, con respecto a la irradiación global incidente en los módulos bifacial, este dependen de la posición, altura, orientación, material reflectante, para optimización del albedo, este parámetro estará asociado al coeficiente de realimentación de energía para el modelo LFSR.

\section{MODELADO DEL SISTEMA EÓLICO: TURBINAS Y OSCILADORES ACOPLADOS}

En el modelado de la turbina se debe considerar en el diseño las dimensiones y geometría configurable de los álabes (telescópicos, replegables, abatibles o compuestos), que capturan la energía cinética 
Tabla 1. Interpretación del modelo realimentado de ERNC en fotovoltaica bifacial.

\begin{tabular}{|c|c|c|c|c|}
\hline \multicolumn{5}{|c|}{$y_{P E R C}(t-1)$} \\
\hline \multicolumn{5}{|c|}{ Superficie Reflectante } \\
\hline \multicolumn{5}{|c|}{$y(t)=\sum_{o p t=1}^{m} w_{o p t} \cdot \sum_{i=1}^{n} c_{i} \cdot x(t)+y_{T}(t-1)+y_{P E R C}(t-1)$, con un componente reflejado $x(t-1)$} \\
\hline \multicolumn{5}{|c|}{ Parámetros aplicables para modelado y optimización del Sistema de Energías Renovables } \\
\hline$w_{m}$ & Descripción del parámetros de modelado & $x_{T}(t)$ & Entrada del modelo & \\
\hline$w_{D I R}$ & Ganancia de la etapa de conversión FV (entrada frontal) & $x_{D I R}(t)$ & Radiación Directa & \\
\hline$w_{D I F}$ & Ganancia de la etapa de conversión bFV (posterior) & $x_{D I F}(t)$ & Radiación Difusa & \multirow{2}{*}{ bFV } \\
\hline$w_{R E F}$ & Ganancia de la etapa de conversión bFV (reflejada) & $x_{R E F}(t)$ & Radiación Reflejada & \\
\hline$w_{T E M P}$ & Atenuación efecto temperatura & $x_{T E M P}(t)$ & Temperatura Ambier & \\
\hline$w_{\lambda}$ & Ganancia/Atenuación por componente espectral solar & $x_{\lambda}(t)$ & Componente Espectı & \\
\hline$w_{\text {opt }}$ & Descripción del parámetro de optimización & $y_{c}(t-1)$ & Realimentación Sele & \\
\hline$w_{s}$ & Peso de formulación de sensibilización & $x b_{P V}(t)$ & Entrada posterior (re & \\
\hline$w_{T}$ & Peso de optimización térmica & $y_{P E R C}(t-1)$ & Fotones realimentad & \\
\hline$w_{S T}$ & Peso de accionamiento del Mecanismo de seguimiento & $y_{E}(t-1)$ & Electrones realiment & \\
\hline$w_{M P P T}$ & Peso del sist. de seguimiento de máxima potencia MPPT & $y_{T}(t-1)$ & Calor residual realim & \\
\hline
\end{tabular}

producen la rotación del eje, donde la optimización de sus parámetros y configuración establece la relación de ganancia. El área barrida por la turbina, que para este modelo será interpretada como registros desplazamiento de energía se corresponden con la configuración Fibonacci, registros para retrasar las entradas en las turbinas secuenciales multi etapas, con $i$ el número de cámaras o etapas. Además de incorporar el efecto de realimentación correspondiente a la energía transferida del último elemento al primero, en el componente inercial de rotación, considerando que en la inicialización se debe establecer las condiciones de velocidad de arranque del aerogenerador AG, en el rango lineal. En la Tabla 2, se presenta una interpretación LFSR generalizada de las turbinas rotacionales.

El esquema puede ser representado en configuración Galois (entrada concurrente delays entre sumadores), retrasando las ganancia parciales de los álabe, en consideración de la respuesta dinámica de una turbina eólica, la acumulación de energía giratoria en el rotor y el comportamiento del eje de transmisión, como un resorte mecánico analizados en [20]. Lo que dota al modelo de un conjunto de términos adaptables para la optimización.

Para estimación de la producción eólica en relación a la intensidad del viento y su dirección se consideran las arquitecturas de ANN que permitan caracterizar el sistema. Las redes Multi Layer Perceptrón - MPL presentan limitaciones al entrenar, el error de la red es muy elevado, según se reporta en [21], por lo que se aplican redes de tercera generación SNN (Spiking Neural Network) y herramientas de serie de tiempo, redes regresivas $\mathrm{y}$ profundas (deep learning) donde se considera transferencia de aprendizaje de parques eólicos establecidos con datos para la extrapolación a sistemas nuevos. En este trabajo se plantea una solución novedosa, basada en aplicar una red de arquitectura coincidente con el modelo físico del sistema eólico realimentado, de forma modular, a fin de simplificar la profundidad de la red y el nivel de abstracción para el proceso de entrenamiento. 
Tabla 2. Interpretación del modelo realimentado de ERNC en turbinas eólicas.

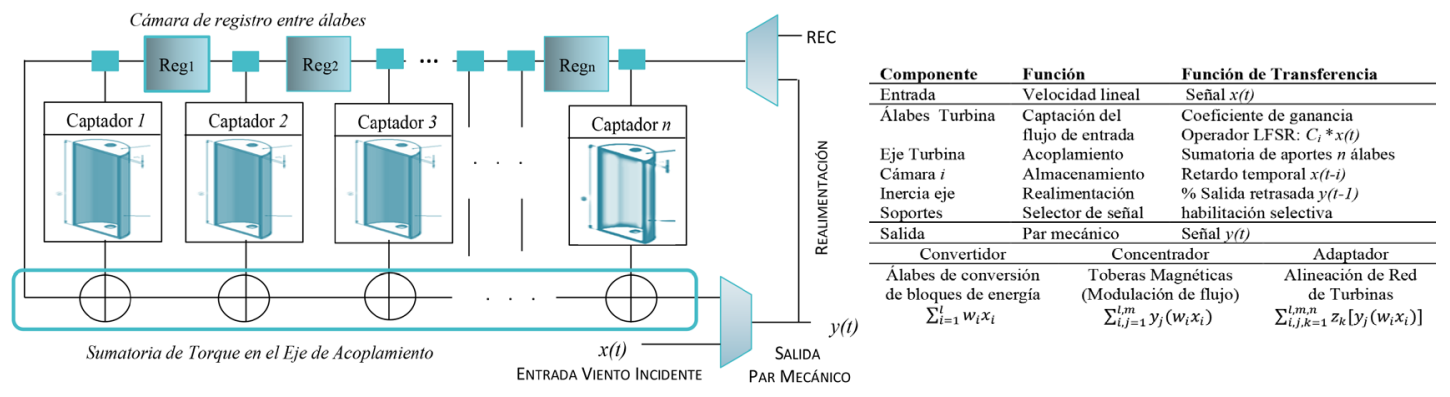

\section{SISTEMA REALIMENTADO LFSR DE ERNC}

Algunos trabajos previos $[22,23]$ plantean soluciones híbridas de energía, en función de estas características se puede programar la asignación porcentual de cada arreglo de convertidores en el sistema: Alternativas de fotovoltaica flotante [24], energías oceánicas [25, 26], eólica [27], así como infraestructura energética implementada, que resulte compatible para la eficiente reutilización en las etapas de almacenamiento de ERNC [22] de modelo circular, a fin de optimizar el diseño por correspondencia entre componentes, a partir del modelado matemáticos de cada subsistema. Dadas las características de intermitencia (dependiente de las condiciones climáticas) de las ERNC, resulta necesario un sistema de almacenamiento para la gestión de la demanda eléctrica [28], diseñados para tiempos cortos de carga y descarga.

De esta manera, es posible plantear en una integración de ERNC, con aporte de cogeneración solar: Fotovoltaica/Termosolar y convertidores de gradiente de temperatura recuperada del ambiente, para almacenamiento térmico directo, considerado en la definición de las ecuaciones descriptiva del esquema ERNC, partiendo de la ecuación (1).

$y_{n}=w_{F V} \cdot i_{S}+w_{T S} \cdot i_{S}+w_{O T E C} \cdot \Delta T+w_{\text {red }} \cdot i_{e}+y_{n-1}(1)$

Siendo $y_{n-1}$ la variable de realimentación de energía residual, este término puede ser generalizado para las diversas formas de energía intermedia, como puede ser potencial eólico no convertido, o cualquier otra forma de energía cinética, que puede ser realimentada al sistema, en tanto que se identifiquen elementos de registro o almacenamiento por etapas.
Un importante aporte de la presente investigación corresponde a la optimización por realimentación, donde se controla las pérdidas del sistema y se recupera la energía residual (térmica en forma de disipación de calor), para mejorar el rendimiento total del sistema de conversión. La estructura LFSR permite modelar los elementos de almacenamiento de energía en el parque eólico o solar y considerar su realimentación en el concepto de sistemas regenerativos. En tal sentido se han estudiado estos elementos y un conjunto de variables alternativas para realimentación, como se presenta en la Tabla 3, donde se identifican las tecnologías de almacenamiento, su aplicabilidad técnica y su correspondencia en el modelo.

Los sistemas de almacenamiento y su potencial de reconversión han sido detallados en [28]. Para minimizar el impacto ambiental, las centrales de almacenamiento térmico, deben ser eficientes en no emitir calor residual al ambiente. Es valioso iniciar con el estudio de potencialidades en materia de infraestructura reutilizable, como en el caso de la termoeléctrica, en base a un esquema conmutado, con válvulas de control. Se plantea un campo termo-solar, las líneas de vapor sobrecalentado, que alimente una caldera solar térmica, sistemas de reducción catalítica de emisiones, sistemas colectores y filtros, así como tanques de almacenamiento, líneas neumáticas (aire comprimido) e hidráulicas, turbinas, recuperadores de calor residual - RCR, en almacenamiento térmico de energía, incorporada como una variable de realimentación, todos estos con el respectivo modelo de actualización con soporte matemático. El primer punto es la reutilización de estos recursos (equipos), a través de una planificación técnica que permita la adaptación de centrales para almacenamiento de ERNC en relación al potencial y 
Tabla 3. Identificación de realimentaciones en el sistema de ERNC.

\begin{tabular}{|c|c|c|}
\hline $\begin{array}{l}\text { Lazo Interno de Realimentación } \\
\quad \text { (a nivel de convertidor) }\end{array}$ & $\begin{array}{l}\text { Lazo Externo de Realimentación } \\
\text { (a nivel de planta) }\end{array}$ & Coef. \\
\hline \multirow{4}{*}{$\begin{array}{l}\text { Fotovoltaica: Recientes investigaciones [29] presentan } \\
\text { la aplicación de un material cristalino, basado en un } \\
\text { tipo de marco orgánico-metálico - MOF, una red de } \\
\text { iones metálicos unidos por moléculas basadas en el } \\
\text { carbono para formar estructuras tridimensionales, estos } \\
\text { pueden formar materiales compuestos, al contener } \\
\text { otras pequeñas moléculas, dentro de sus estructuras, } \\
\text { que le permiten capturar energía, los investigadores } \\
\text { expusieron el material a la luz UV, encontrando que } \\
\text { la energía potencial puede almacenarse durante largos } \\
\text { períodos de tiempo a temperatura ambiente y se puede } \\
\text { liberar a demanda. }\end{array}$} & $\begin{array}{l}\text { Recuperación de calor en circuitos de } \\
\text { refrigeración de aplicaciones fotovoltaicas } \\
\text { [30], que puede ser reutilizado como energía } \\
\text { térmica regenerativa, en sistema de cogeneración } \\
\text { termo-solar fotovoltaico. }\end{array}$ & $w_{C G}$ \\
\hline & $\begin{array}{l}\text { Ultra-condensadores, por sus características } \\
\text { para la etapa transitoria de optimización del } \\
\text { arreglo fotovoltaico. }\end{array}$ & $w_{E}$ \\
\hline & Almacenamiento de vapor [31]. & $\mathrm{w}_{\mathrm{V}}$ \\
\hline & i espacios reducidos como sales term & $w_{T S}$ \\
\hline $\begin{array}{l}\text { Esta tecnolog } \\
\text { describir regi } \\
\text { el convertido }\end{array}$ & \multirow{3}{*}{$\begin{array}{l}\text { En el caso de almacenamiento térmico con } \\
\text { cambio de estado, la cantidad de energía } \\
\text { almacenada corresponde al modelo detallado en } \\
\text { [33], en relación a la masa de almacenamiento } \\
\text { y los coeficientes térmicos de los materiales en } \\
\text { el cambio de fase. En [35] se han propuesto un } \\
\text { sistema de almacenamiento térmico denominado } \\
\text { Batería Carnot, el cual consiste en integrar } \\
\text { tanques de sales fundidas (como en las plantas } \\
\text { de concentración solar de potencia - CSP), este } \\
\text { tipo de tecnología utiliza la electricidad de ERNC } \\
\text { para la fundición de sales, su almacenamiento, } \\
\text { en forma de energía térmica y la reconversión a } \\
\text { energía eléctrica, a través de turbinas de vapor, } \\
\text { cuando se requiera. En estas aplicaciones de } \\
\text { requiere un convertidor de almacenamiento } \\
\text { térmico (Turbina C.A.T). }\end{array}$} & \multirow{3}{*}{$w_{R C R}$} \\
\hline $\begin{array}{l}\text { OTEC, Convertidores por gradiente de temperatu } \\
\text { oceánico [34], sistemas en circuitos cerrados realimentad }\end{array}$ & & \\
\hline \multirow{3}{*}{$\begin{array}{l}\text { Élica: Realimentación de aire entre la distribución de } \\
\text { elementos, siendo los registros, el área de barrido de } \\
\text { los álabes. De esta manera, el avance de giro se modela } \\
\text { como un registro desplazamiento, con un transitorio y un } \\
\text { régimen permanente, en el que se estabilizan los aportes } \\
\text { colaborativos de realimentación, del sistema regenerativo. } \\
\text { Para alcanzar una estabilidad en el comportamiento } \\
\text { dinámico, se modela el sistema de almacenamiento del } \\
\text { parque eólico, alineación óptima y realimentación, a fin } \\
\text { de mantener las condiciones de operación. } \\
\text { Turbinas de Desplazamiento de Bloques de Energía: } \\
\text { Los elementos rotativos, que presentan cámaras de aire o } \\
\text { elementos que se desplazan y realimentan, tal es el caso } \\
\text { de sistemas undimotriz y otras tecnologías en el sector. }\end{array}$} & & \\
\hline & $\begin{array}{l}\text { En [1] se presentó un esquema LFSR con } \\
\text { almacenamie@ @ nto de energía mecánica/ } \\
\text { neumática, basado en múltiples etapas de aire } \\
\text { comprimido. }\end{array}$ & $w_{A C}$ \\
\hline & $\begin{array}{l}\text { Bombeo Hidráulico multi-etapa, a través de } \\
\text { tanques de almacenamiento, bombas hidráulicas } \\
\text { y realimentación al sistema. }\end{array}$ & $w_{H}$ \\
\hline$\frac{d=1}{\text { C.A.E. }}$ & \multicolumn{2}{|c|}{$\begin{array}{l}\text { Los parámetros para la selección corresponden a densidad } \\
\text { de energía/potencia y velocidad de almacenamiento, } \\
\text { vida útil, asociados a los ciclos de cargar y descargar } \\
\text { sin sufrir pérdidas de rendimiento significativas, y } \\
\text { eficiencia energética. De esta manera, se definen dos } \\
\text { estados de estabilidad del sistema, inicialización con } \\
\text { condiciones externas y régimen de realimentación. } \\
\text { El modelo puede ser aplicada a través de estudio de } \\
\text { perfiles de potencial termo-gráfico, así como tecnología } \\
\text { de conversión termodinámica para aprovechamiento de } \\
\text { aporte de calor regenerativo al sistema, bajo criterios } \\
\text { ambientales. }\end{array}$} \\
\hline
\end{tabular}


alternativas de configuración (Ver Tabla 4), todo esto con el propósito de mitigar el impacto ambiental, a través de la reutilización, reciclaje, remediación ambiental y desarrollo sostenible.

Partiendo de la necesidad de migración de las centrales de energía termoeléctricas a ERNC y los conceptos estudiados es importante diseñar sistemas reconfigurables, aplicando acoplamientos mecánicos o magnéticos programables para etapas de energías renovables, actualización del hardware y reconfiguración dinámica, en relación a los datos de las condiciones climáticas, características del sistema y emisiones, a fin de establecer criterios de configuración óptima y la reutilización de la infraestructura existente, así como la adaptación de los puestos de trabajo a las nuevas tecnologías. En el caso de cada tecnología se requiere un estudio del impacto ambiental, considerando los efectos sobre la biodiversidad. Con especial atención a las emisiones de calor residual al ambiente que deben ser controladas, a través de tecnologías de aislamiento térmico y optimización termodinámica.

Es por ello, se debe adaptar la arquitectura a la potencialidad en ERNC con un enfoque de matriz conmutada para configuración dinámica, almacenamiento y realimentación selectiva,

Tabla 4. Alternativas de configuración del sistema de ERNC

\begin{tabular}{|c|c|c|c|c|c|}
\hline & Tecnologías de ERNC & Potencial Estimado ${ }^{5}$ & Almacenamiento & Realimentación & I.A.1 \\
\hline \multirow{5}{*}{$\frac{\overline{3}}{\tilde{\sigma}}$} & Termo-solar & Irradiancia (W/m2) & Térmico RCR ${ }^{2}$ & E. Térmica & - \\
\hline & Fotovoltaica & $6.32 \mathrm{KW} / \mathrm{m} 2 /$ día & Luminiscencia, UC & PERC, Bifacial & {$[36]$} \\
\hline & \multicolumn{2}{|c|}{$\begin{array}{l}\text { De los perfiles de incidencia de energía para la región } \\
\text { estudiada, destaca una radiación solar, lo que representa una } \\
\text { alto potencial de energía, para el aporte al esquema híbrido, } \\
\text { definiendo alternativas de cogeneración solar (fotovoltaica/ } \\
\text { termosolar). Igualmente, se observan altos niveles de incidencia } \\
\text { solar en reservas naturales y glaciares, áreas que requieren } \\
\text { ser protegidas de la radiación directa, donde es posible } \\
\text { plantear un arreglo de re-direccionamiento inteligente [32]. }\end{array}$} & \multicolumn{3}{|c|}{$\begin{array}{l}\text { Optimización. } \\
\text { - Filtrado/concentración por longitud de onda. } \\
\text { - Control y RCR de temperatura del módulo FV. } \\
\text { - Algoritmo MPPT a la salida del convertidor. } \\
\text { - Eficiencia cuántica, PERC. } \\
\text { - Recuperación de radiación reflejada en módulo bifacial. } \\
\text { - Agro-fotovoltaica para recuperación de espacios. }\end{array}$} \\
\hline & Material Termo-Eléctrico & Temperatura $\left({ }^{\circ} \mathrm{C}\right)$ & \multirow{2}{*}{$\begin{array}{l}\text { MOF [29] } \\
\text { Nueva Tecnología }\end{array}$} & \multirow{2}{*}{ Calor Residual } & - \\
\hline & Cogeneración Solar (E+T) & - & & & \\
\hline \multirow{3}{*}{ 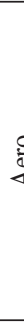 } & Turbina Eólica (E. Cinética) & $4.5 \mathrm{~m} / \mathrm{s}$ & CAES $^{3}$ & Aire Comprimido & {$[37]$} \\
\hline & $\begin{array}{l}\text { Un factor importante es la altura, } \\
\text { donde los aerogeneradores } \\
\text { volantes, pueden tener mejores } \\
\text { prestaciones y flexibilidad. }\end{array}$ & $\begin{array}{l}\text { Tecnología de Turbina: } \\
\text { - Eólica Horizontal. } \\
\text { - Eólica Vertical. } \\
\text { - Eólica Aérea } \\
\text { - NTE, VIV, Resonancia. }\end{array}$ & \multicolumn{3}{|c|}{$\begin{array}{l}\text { Optimización } \\
\text { - Técnicas de protección a las aves (formadores de perfil). Concentrador } \\
\text { de viento (Toberas). Geometría Configurable. Levitación magnética } \\
\text { MagLev. Turbinas colaborativas. Realimentación almacenada } \\
\text { (CAES). }\end{array}$} \\
\hline & Vibración (piezoeléctrico) & - & - & - & - \\
\hline & Sistemas Aero-térmicos & $\Delta \mathrm{T}\left({ }^{\circ} \mathrm{C}\right)$ & Térmico RCR $^{2}$ & Calor Ambiental & - \\
\hline \multirow{4}{*}{ 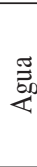 } & Hidroeléctrica & & Reserva $\left(\mathrm{m}^{3}\right)$ & Bombeo & Hábitat \\
\hline & OTEC (gradiente térmico) & $\Delta \mathrm{T}\left({ }^{\circ} \mathrm{C}\right)$ & Térmico RCR $^{2}$ & Agua, E. Térmica & \multirow{3}{*}{$\begin{array}{c}\text { Hábitat } \\
\text { En Estudio }\end{array}$} \\
\hline & Undimotriz (E. Cinética) & $15 \mathrm{~s}$ & Volante de Inercia & - & \\
\hline & Undimotriz (E. Potencial) & $1.2 \mathrm{~m}-2.3 \mathrm{~m}$ & Resortes Radioid. & - & \\
\hline \multirow{3}{*}{ ర్ర } & Geotérmica & $\Delta \mathrm{T}\left({ }^{\circ} \mathrm{C}\right)$ & - & \multirow{2}{*}{ Calor Residual } & Alto \\
\hline & Biomasa & P. calorífico $(\mathrm{KJ} / \mathrm{g})$ & Biomasa & & Alto \\
\hline & RSU (Residuos Sólidos) & P. calorífico $(\mathrm{KJ} / \mathrm{g})$ & Residuos & & Alto \\
\hline
\end{tabular}

1 I.A. Impacto Ambiental.

2 Recuperación de calor (RCR): Líneas hidráulicas (tanques, bomba, válvulas) disponibles.

3 UC: Ultra-condensadores, CAES: Sistemas de almacenamiento de Aire Comprimido. Líneas de aire comprimido Disponibles.

${ }^{4}$ Undimotriz (E.C): Periodo (s). Undimotriz (E.P): Amplitud de la ola (m). Eólica: Velocidad de viento (m/s).

5 Potencial Estimado con Herramienta: Explorador Solar. 
coincidiendo con el esquema LFSR [10-12] (Linear Feedback Shift Register). Esto orientado capas paralelas de elementos de configuración del sistema, donde la creciente demanda de digitalización de la infraestructura energética da espacio a desarrollos basados en tecnologías FPGA (Field Programmable Gates Array), como dispositivos inteligentes para la definición de parámetros de control para máxima eficiencia y arquitectura dinámica de los arreglos en ERNC.

\section{METODOLOGÍA}

Se estudia un sistema de ERNC para establecer la correspondencia con un esquema híbrido LFSR de concatenación de convertidores y definir las variables de optimización.

Método de modelado LFSR para sistemas realimentados de energía renovables, está basado en el reconocimiento de patrones estructurales, a fin de establecer una correlación, por lo que se ha realizado un análisis de los sistemas de energías renovables, a fin de entender su comportamiento e identificar coincidencias estructurales (ver Tabla 5).

En el método de optimización se planteó la fijación de la meta para el sistema, la adaptación de parámetros altura y radio por estructuras telescópicas, posición de comentas eólicas flotantes, ángulo de alineación, ángulo de pitch., para alcanzar la potencia de salida en relación a las nuevas condiciones. Tecnología de soportes de levitación magnética MagLev para disminuir pérdidas de fricción, equivale en $\mathrm{FV}$ a materiales para evitar la recombinación de electrones.

Seguidamente, se han tabulado estos parámetros físicos para la definición de una ecuación que permite describir la relación entre los componentes del modelo. El operador LFSR como subred ANN, modela cada etapa del sistema, luego las etapas son concatenadas y realimentadas.

Método de obtención de los coeficientes, se plantea un método semi-empírico basado en los parámetros medidos o estimados del sistema, que permita el entrenamiento de la red neuronal, de forma modular, lo que representa establecer la relación por módulos, a fin de disminuir el nivel de abstracción, así mismo acceder a los coeficientes y parámetros físicos del sistema a optimizar. Se logró una metodología de modelado, donde la configuración de los registros desplazamiento se define por el comportamiento del sistema físico de conversión y permite definir la arquitectura de la ANN más apropiada para cada etapa, sea de estructura concurrente MPL o recurrente RNN.

Tabla 5. Correspondencia entre el modelo LFSR y turbina eólica generalizada.

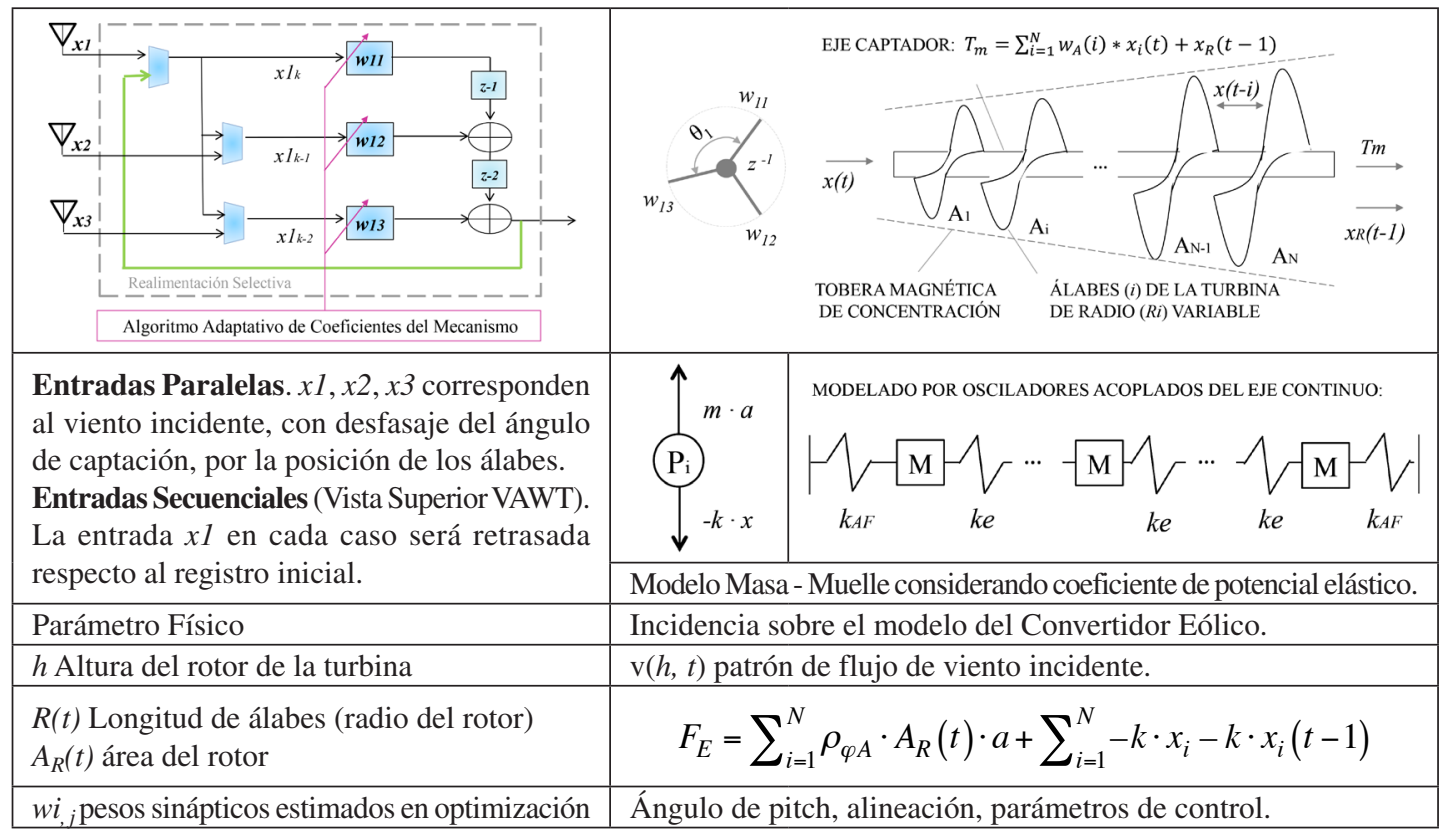


Método de optimización. Inicialmente se definen los módulos de optimización: Concentración (toberas magnéticas /óptica), elementos de modulación de patrón incidente y alineación (patrón reflejado), control de pérdidas del sistema, filtros (componentes de radiación UV, filtros de aire en turbinas), protectores de la fauna local, nuevas tecnologías emergentes, a su vez el modelo permite encontrar la relación de rendimiento del sistema, sobre ésta se aplican métodos de optimización matemáticos y se hallan los máximos, a partir de los targets de eficiencia óptima del sistema, se pueden estimar los coeficientes optimizados por la red neuronal será la encargada de adaptar los coeficientes y generar las señales de accionamiento del hardware, para optimizar las etapas del sistema (ver Tabla 6).

Seguidamente, se define las etapas de modelado: Etapa externa de optimización por coeficientes adaptativos. Etapa del modelo neuronal, coeficientes de conversión por capa paralelas (neurona de la capa oculta) y voltaje de activación (umbral / bias). El modelo se ha orientado al tratamiento de subredes independientes en relación a las etapas del sistema físico (Ver Figura 1), con la interpretación de los pesos sinápticos y los coeficientes técnicos. Esto permite desactivar capas independientes (layout) para la simplificación del proceso de optimización dinámica, hallar los pesos sinápticos y con un cálculo reversible, se puede analizar el rendimiento de cada factor de optimización.

En el esquema se ilustra de forma resumida las etapas del arreglo de ERNC (combinación Fotovoltaica - Eólica), los parámetros que pueden ser adaptados (externos) y el modelo simplificado con los parámetros fijos (internos del semiconductor). Los datos de irradiancia y generación fotovoltaica permiten entrenar la red neuronal MPL interna, en tanto que la red externa (recurrente), se define a partir de los valores optimizados de potencia fotovoltaica. Es de hacer notar, que en la etapa cuántica, se han considerado los coeficientes fijos, definidos como coeficientes de la tecnología. Estos pueden ser estimados por dos métodos: Redes neuronales MPL (simplificado) o el modelo secuencial con redes dinámicas.

\section{Caso de Estudio: Estimación de parámetros}

Para el caso de instalaciones fotovoltaicas la herramienta permite estimar la producción eléctrica, basada en el modelo de radiación solar y modelo de

Tabla 6. Método de modelado neuronal del sistema de ERNC.

\footnotetext{
Modelo básico de la relación de conversión fotovoltaica y eólica.

Estimación de potencial del Arreglo Fotovoltaico.

Estimación de potencial Eólico.

Entrenamiento de una red neuronal multicapa para modelado de las plantas.

Reconocimiento de patrones y términos componentes del modelo generalizado.

Comparación estructural de sistemas de conversión de energías renovables.

Identificación de correspondencia entre términos del modelo.

Propuesta de optimización basada en modelo de realimentación.

Extrapolación del esquema a los sistemas de almacenamiento de ERNC.

Definición de coeficientes de optimización aplicables a los sistemas de ERNC.

Sistema Híbrido: El potencial de cada fuente de ERNC se complementa por las horas pico de máxima potencia.
}

Identificación de Realimnentación:
En el caso de Eólica, la interacción entre
los álabes o las turbinas alineadas se
pueden modelar como realimentaciones,
en un comportamiento de bi-estabile, en
régimen permanente.
En el caso de Fotovoltaica Bifacial (bPV),
el factor de bifacialidad corresponde al
coeficiente de realimentación.
En el sistema integrado, la energía de las
etapas de almacenamiento de ERNC
son realimentadas al sistemas de ERNC.

\begin{tabular}{|l|l|}
\hline \multicolumn{1}{|c|}{ Concentrador de radiación solar } & \multicolumn{1}{c|}{ Toberas Magnéticas de concentración eólica } \\
\hline Modificador del haz incidente sobre panel de conversión solar & $\begin{array}{l}\text { Modulador eólico de flujo axial, radial y toroidal por turbinas } \\
\text { concatenadas concéntricas. }\end{array}$ \\
\hline Ganancia del panel fotovoltaico. & Ganancia de álabes captadores: Radio, Altura, ángulo, perfil. \\
\hline PERC Realimentación de fotones. & Realimentación de energía cinética entre álabes e Inercia del eje. \\
\hline Tecnología Bifacial. & Realimentación entre turbinas, control de alineación. \\
\hline Electrones realimentados. & Realimentación de aire comprimido. \\
\hline Repetidora selectiva (heliostato). & Arreglo de Turbinas Colaborativas en distribución fractal. \\
\hline Guía de Onda / Fibra óptica para concentrar radiación. & Líneas Neumáticas del sistemas de realimentación dedicado. \\
\hline Filtro de componentes no eficaces, protección de áreas. & Protección de fauna, sensores y diseños no invasivos. \\
\hline
\end{tabular}




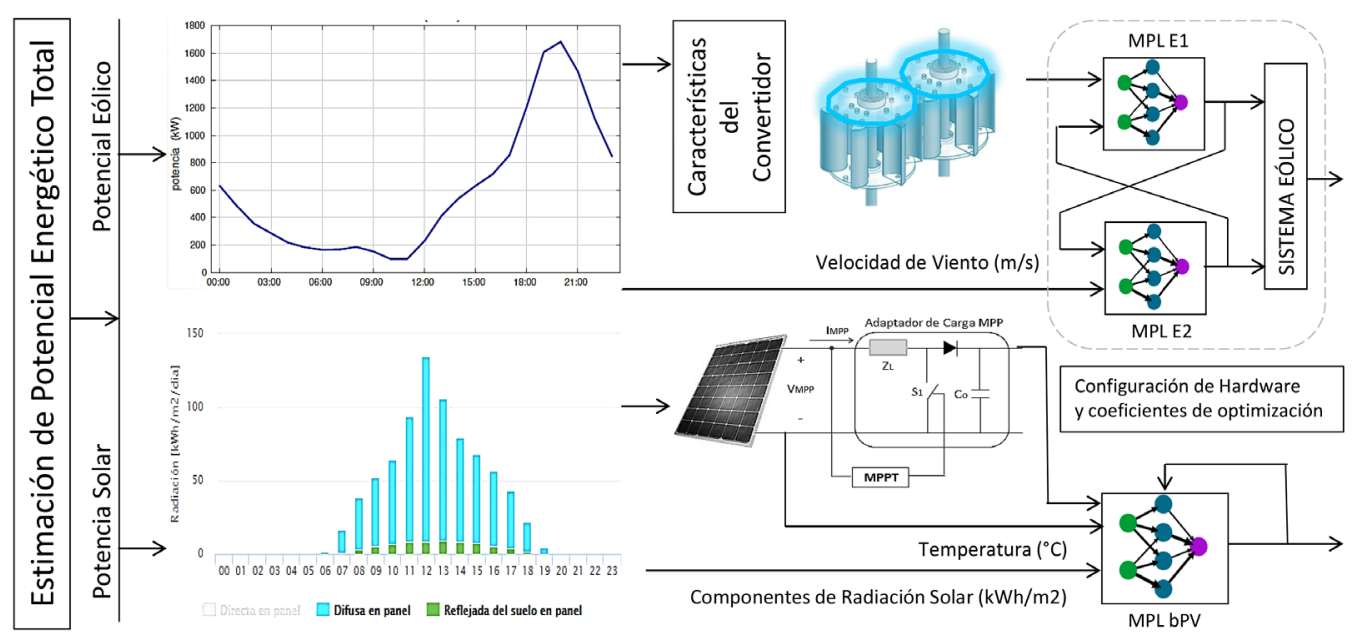

Figura 1. Método de optimización por realimentado y coeficientes adaptativos.

generación fotovoltaica, en relación con la superficie instalada, eficiencia del panel fotovoltaico y tipo de arreglo, obteniendo el reporte de la Tabla 7. Estos resultados permiten identificar el ángulo de incidencia como un coeficiente adaptativo. Este puede ser adaptado en el sistema, a partir del modelo desarrollado, con la configuración dinámica del arreglo fotovoltaico, a través de tecnología FPGA. Así mismo, la incorporación de RCR, para el control de temperatura del módulo (panel fotovoltaico), a fin de aumentar su eficiencia.

Este modelo aplica las especificaciones técnicas del módulo PV seleccionado en el diseño de la planta (arreglo fotovoltaico), donde se definen características como potencia del panel, número de celdas, coeficiente de temperatura, eficiencia, esquema de conexión, inversor y parámetros eléctricos como voltaje y corriente de máxima potencia $\mathrm{V}_{\mathrm{MPP}}$, $\mathrm{I}_{\mathrm{MPP}}, \mathrm{V}_{\mathrm{oc}}, \mathrm{I}_{\mathrm{sc}}$.

En fotovoltaica se puede diseñar un optimizador para cada variable de entrada: Radiación incidente (clase de radiación y longitud de onda) y temperatura, así como un optimizador en las variables de salida (MPPT en la carga del SFV), además del coeficiente de realimentación de energía, a través de variables de composición fractal y operadores convolucionales. En el caso del sistema de ERNC, cada convertidor presenta una descripción LFSR, considerando la variable de conversión directa y componentes espectrales, concatenados en un esquema con realimentación de la variable de salida, estos compatibles entre los módulos.

La extrapolación de esta arquitectura descriptiva, puede ser aplicada para el modelado de tecnología fotovoltaica bifacial, sistemas de turbinas de entradas paralelas, multi-etapas y secuenciales, donde se reconoce la estructura LFSR, tal es el caso de los elementos eólicos y otros módulos de conversión rotativos. Es importante resaltar, que la arquitectura de la ANN modeladora y optimizadora (de control), tienen un modelo matemático similar (con estructura LFSR). Es decir, se hacen coincidir los pesos sinápticos del entrenamiento de la red, con los coeficientes del modelo desarrollado. Se obtiene así un modelo concatenado $\mathrm{A}(\mathrm{a}(\mathrm{x}))$, que permite reproducir el modelo y optimizarlo. Los datos de entrada corresponden a la irradiancia (radiación directa, radiación difusa y radiación reflejada) y temperatura.

Para la definición del modelo se estudia la interrelación de los parámetros, una vez reconocido el patrón estructural del sistema, sus componentes y las redes neuronales modeladoras, se estiman los coeficientes que describen la relación de variables de entrada y salida del arreglo de conversión. El diseño modular permite establecer etapas funcionales (concatenadas), tales como concentración de energías renovables previas al convertidor, relación de parámetros, selectividad y direccionamiento para optimización del arreglo, lo que corresponde a subredes o capas de la DNN. Luego, se definen 
Tabla 7. Estimación de potencial de ERNC fotovoltaica y eólica para entrenamiento del modelo.

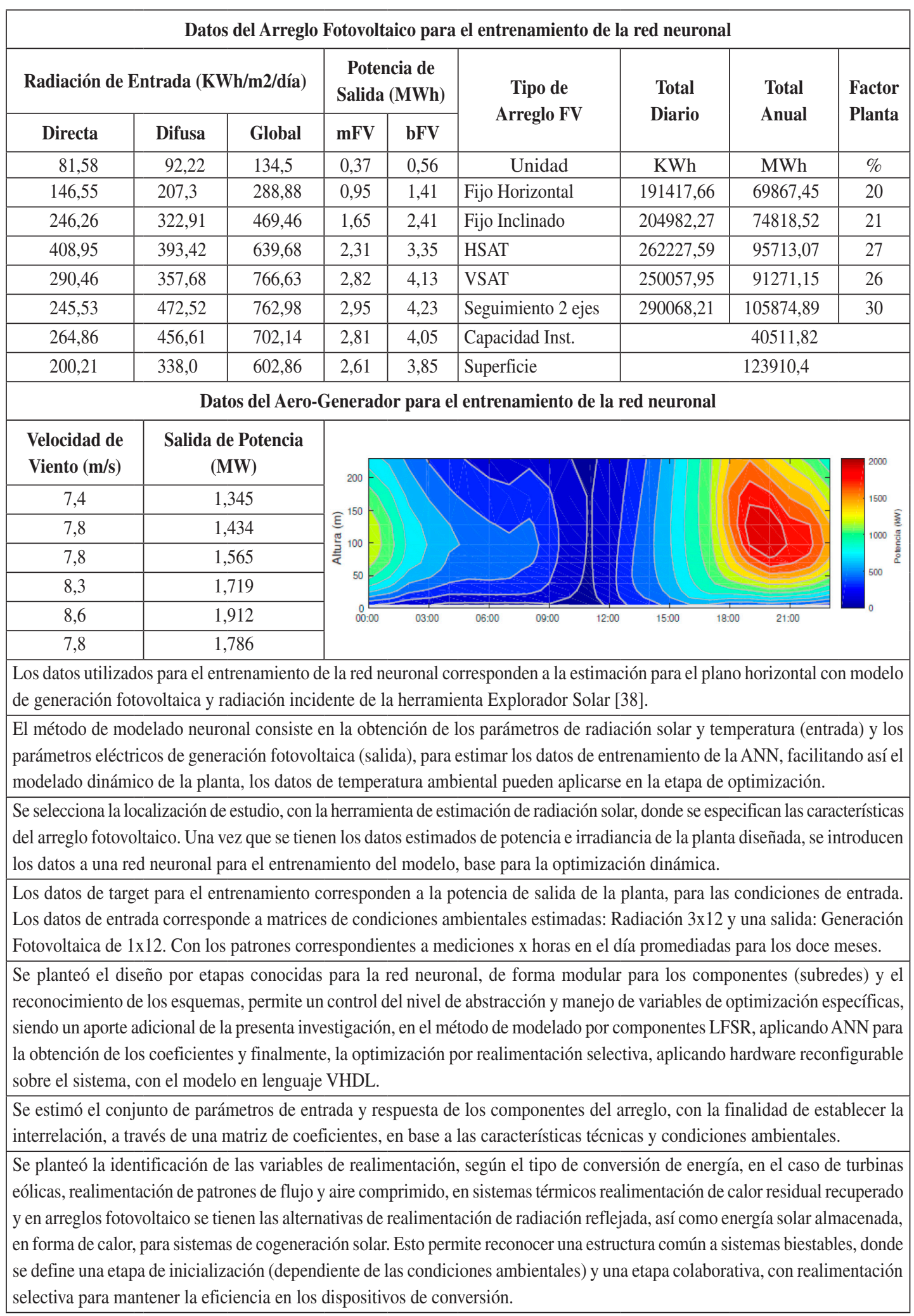


los targets correspondientes a la salida óptima, con estos se estiman los coeficientes de realimentación, correspondientes a pesos adaptativos de la red recurrente, en la capa externa, que permita la optimización por realimentación ponderada.

\section{ALTERNATIVAS DE OPTIMIZACIÓN Y DESCRIPCIÓN EN EL MODELO LFSR}

Para el caso de fotovoltaica, el convertidor optimizado deberá integrar los términos de aprovechamiento de radiación difusa y radiación reflejada $w_{R}$, para los modelos bifaciales: $y_{n}=w_{D} \cdot i_{s}+w_{R} \cdot i_{s}+y_{n-1}$ $\mathrm{o}$ arreglos concentradores $w_{c}$, con optimización en diversas etapas: $y_{n}=w_{C} \cdot i_{s}+w_{P V} \cdot i_{s}+w_{M P P} \cdot i_{s}+$ $y_{n-1}$. Luego interpretando las etapas de conversión de la energía solar captada para almacenamiento, se puede re-expresar de la forma generalizada de la ecuación (2).

$y_{n}=\sum_{i=1}^{m} w_{c i} \cdot \delta_{c}+\sum_{j=1}^{n} w_{\text {convj }} \cdot i_{s}+\sum_{k=1}^{r} b_{k} \cdot y_{n-1}$

Donde $w_{c} \cdot \delta_{c}$ corresponde al término selectivo de concentración por componente de radiación (tomada en cuenta para el modelo ANN), sensibilización espectral y clase de incidencia solar, que se presenta como la etapa óptica, previa a la conversión. El término $w_{c o n v} \cdot i_{s}$ de conversión eléctrica y $y_{n-1}$ la realimentación selectiva con pesos ponderados $b_{k}$ por componente de energía residual regenerativa. El modelo del sistema con realimentación de energía, debe cumplir criterios ambientales: Cero residuos, máxima eficiencia energética, cero emisiones de calor residual, sin modificación las propiedades químicas del agua (circuito cerrado), control de interferencia, estudio de impacto electromagnético (en redes de potencia y sobre la fauna), ciclos de recuperación (descanso/desacoplado) y programas de reciclaje.

Algunos parámetros han sido considerados fijos a partir de los datos técnicos de los módulos, como son la eficiencia del panel $\mathrm{FV}$, la concentración de radiación solar incidente en $\mathrm{W} / \mathrm{m}^{2}$, el índice de rendimiento térmico y porcentaje de eficiencia del inversor, sin embargo las técnicas de optimización diseñadas permiten obtener mejoras en el comportamiento del sistema fotovoltaico y la eficiencia total de la instalación. Ambos convertidores pueden presentar realimentación, en fotovoltaica se puede identificar en los paneles bifaciales y módulos PERC, en tanto que en turbinas en el diseño de las aspas para captación de flujo residual realimentado para conversión eficiente de energía, estas variables de optimización serán adaptadas aplicando el modelo de control para la estimación del coeficiente óptimo.

Para el modelo de fotovoltaica bifacial, la radiación reflejada corresponde a un entrada retrasada en $t-1$, que es realimentada para su aporte en la salida de potencia total, para esto se ha considerado el número de neuronas de la capa oculta en relación a los componentes de radiación directa + difusa + reflejada (según el plano del arreglo FV estimada por el sistema). La simplificación viene dada por el manejo de etapas de forma paralela, que pueden ser modeladas con una red neuronal MPL, a fin de obtener la matriz de coeficientes del modelo, como alternativa al entrenamiento de red dinámica. Para este caso, se incorpora una capa oculta de función de activación no lineal, que modela el comportamiento del esquema LFSR, donde se pueden mapear los parámetros de forma directa.

Optimizaciones: Concentradores selectivos o inteligentes, mecanismos MagLev para soportes o mecanismos articulados, aislamiento térmico y recuperación de calor para reutilización de energía residual. Estos comprenden una etapa ponderada en la etapa de pre-conversión, captación y realimentación en la etapa posterior a la conversión. Con esto se logra un sistema más eficiente, control de pérdidas asociadas (como fricción) y mayor vida útil de los componentes (con filtrado de componentes espectrales), logrando así estandarizar el método de optimización por modelado LFSR.

Se debe considerar el comportamiento de la señal realimentada, que se presenta en la dinámica de los componentes de la señal de entrada, proveniente del Modelo de Radiación Incidente ( $I_{P O A}$ ) sobre el panel corresponde al aporte de los componentes de radiación solar [38]. Para el caso del panel bifacial se puede expresar de la forma (3):

$$
I_{b F V}=I_{\text {frontal }}+I_{\text {posterior }} \rightarrow\left\{\begin{array}{c}
I_{\text {frontal del panel }}=D I R \\
I A M+D I F_{\text {inc }}+D I F_{\text {suelo }} \\
I_{\text {reflejada }}=(D I R \cdot I A M+ \\
\left.D I F_{\text {inc }}+D I F_{\text {suelo }}\right) \cdot \text { Albedo }
\end{array}\right.
$$

Siendo IAM el ángulo de incidencia, como un factor de ponderación de la radiación directa y el albedo 
el porcentaje de radiación que cualquier superficie refleja respecto a la radiación que incide sobre ella. Considerando la radiación reflejada, como una realimentación de la radiación incidente ponderada por el albedo, de la forma (4):

$$
I_{b F V}=\begin{gathered}
D I R \cdot I A M+D I F_{\text {inc }}+D I F_{\text {suelo }}+ \\
\left(D I R \cdot I A M+D I F_{\text {inc }}+D I F_{\text {suelo }}\right) \cdot \text { Albedo }
\end{gathered}
$$

En relación a la aproximación al modelo realimentado, se presenta la deducción:

Donde cada coeficiente corresponde a un operador LFSR concatenado en función de las dimensiones $i, t$, así la operación con los coeficientes y señales del modelo, corresponden a la convolución de un esquema LFSR:

En una primera aproximación se consideran coeficientes fijos.

$$
y_{i}(t)=\sum_{i, t=1}^{m} c_{i} \cdot x_{i}(t)+b_{i} \cdot y_{i}(t-1)
$$

Luego, se definen como coeficientes dinámicos.

$$
y_{i}(t)=\sum_{i=1}^{m} c_{i}(t) \cdot x_{i}(t)+b_{i}(t) \cdot y_{i}(t-1)
$$

En el modelo se establece la operación con coeficientes que dependen de las dimensiones $\mathrm{i}, \mathrm{t}$ con estructura LFSR:

$$
y_{i, t}(x)=\sum_{x, t=1}^{m, n} c_{i, t} \cdot x_{i}(t)+\cdot b_{i, t} \cdot y_{i, t}(t-1)
$$

\section{RESULTADOS}

El modelo desarrollado presenta operadores en configuración LFSR (con habilitadores lógicos) y la adaptación de los coeficientes del sistema. En las configuraciones estudiadas se observa un compromiso entre el nivel de redundancia, eficiencia y flexibilidad en la optimización. Esto a través del esquema de configuración dinámica (basada en control entrenado sobre HW), extendiendo el concepto de hardware reconfigurable al arreglo de la matriz energética.

Se seleccionan los datos de entrada para radiación: Directa, Difusa y Global (kWh/m²/día) y la salida en potencia (MWh), la arquitectura de la red corresponde a tres neuronas en la capa oculta y una neurona en la capa salida. Es importante mencionar que se realizaron pruebas de ensayo para calcular el número de neuronas óptimas para la red modeladora y la función de activación, considerando los parámetros de saturación característicos de los módulos fotovoltaicos. Los resultados obtenidos en el entrenamiento neuronal para el modelo de fotovoltaica monofacial - (ver Tabla 8.a) y bifacial (Ver Tabla 8.b).

La selección del número de neuronas en función del modelo, permitió un error medio de 0,03 en mPV y 0,04 en bPV, observando que converge en 150 épocas. Los parámetros físicos y su relación con los componentes de la red neuronal modeladora, se han identificado en la Tabla 9, en los que se reconocen las variables de realimentación y coeficientes de optimización dinámica, en relación a las técnicas disponibles.

En primer lugar se hicieron ensayos, asignando un número de capas y neuronas en relación al sistema físico. Para la ANN modeladora del sistema eólico, se seleccionó una red de 2 capas ocultas, para el modelado de la etapa mecánica de conversión de potencial eólico, siendo la entrada velocidad lineal del flujo de viento $F_{R}=\sum_{i=1}^{N} m \cdot a+\sum_{i=1}^{N}-k \cdot x(t-1)$,

Tabla 8.a. Modelado neuronal del arreglo fotovoltaico mPV.

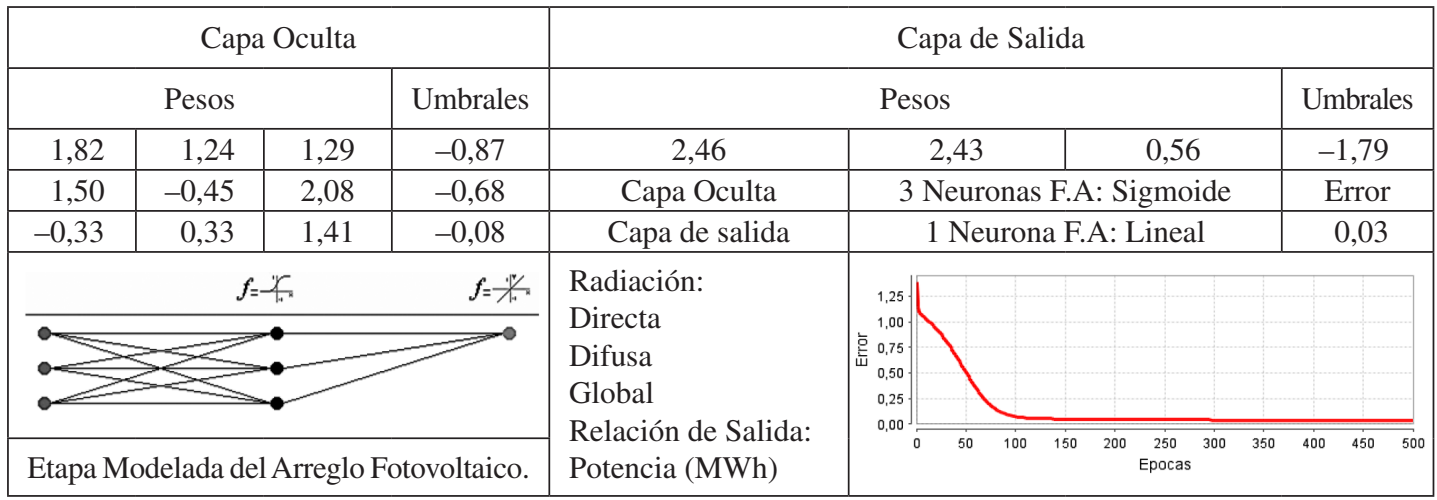


Tabla 8.b. Modelado neuronal del arreglo fotovoltaico bPV.

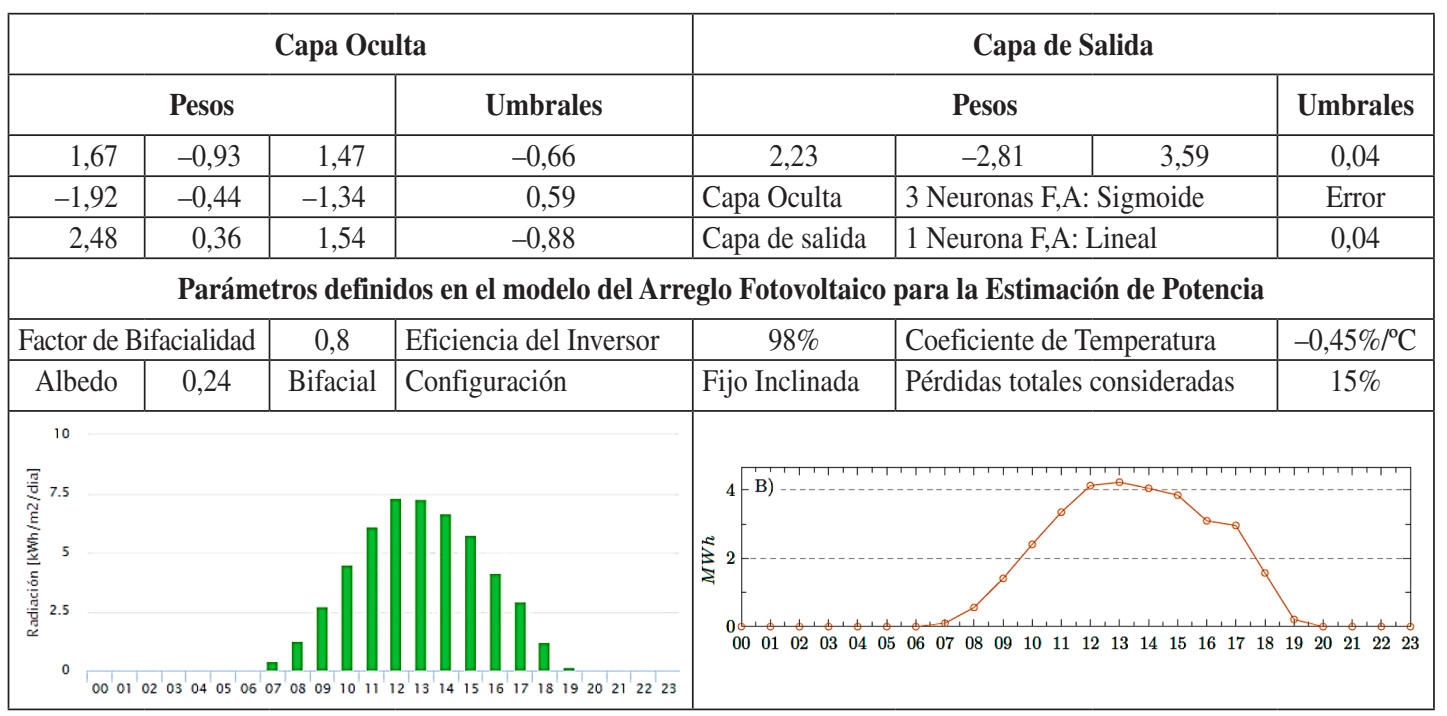

Tabla 9. Etapas del modelo del arreglo fotovoltaico.

\begin{tabular}{|c|c|c|c|}
\hline Etapa/Nivel & Coeficientes & Parámetros Físicos del Arreglo Fotovoltaico & Correspondencia en el modelo \\
\hline \multirow{4}{*}{ Cuántica } & \multirow{4}{*}{ Fijos } & Coeficiente de Absorción & \multirow{2}{*}{ Parámetros Internos del material } \\
\hline & & Concentración de Dopante en el Semiconductor $(\phi)$ & \\
\hline & & ${ }^{1}$ Banda de Valencia (Ev), Banda de Conducción (Ec) & Umbrales por capa FV. \\
\hline & & Flujo de electrones emitidos (corriente en capas) & Sumatoria de aportes \\
\hline \multirow{6}{*}{$\begin{array}{l}\text { Convertidor } \\
\text { Fotovoltaico }\end{array}$} & \multirow{5}{*}{ Red MPL } & Eficiencia de Conversión $(\%)$ & \multirow{3}{*}{$\begin{array}{l}\text { Pesos de la capa oculta } \\
\text { representa ganancia en panel FV } \\
\text { (signo según su aporte) }\end{array}$} \\
\hline & & ${ }^{3}$ Coeficiente de temperatura (pérdidas) & \\
\hline & & Parámetros eléctricos: $F F, V_{M P P}, I_{M P P}, V_{O C}, I_{S C}$. & \\
\hline & & Eficiencia del Inversor & Pesos de la capa de salida \\
\hline & & ${ }^{1}$ Banda de Energía prohibida $(E g)$ & Función de Activación (Sigmoide) \\
\hline & Red Recurrente & Realimentación simplificada del modelo & ${ }^{2}$ Error del modelo de la red MPL \\
\hline \multirow{5}{*}{$\begin{array}{l}\text { Optimización } \\
\text { Dinámica }\end{array}$} & \multirow{5}{*}{ Adaptativos } & MPPT a la salida del arreglo & PWM del circuito de salida \\
\hline & & Control de Temperatura & Coeficiente de Refrigeración \\
\hline & & Irradiancia (Seguimiento Solar) & IAM ángulo de incidencia \\
\hline & & Óptica (concentración externa) & Factor del lente (opcional) \\
\hline & & Configuración dinámica del arreglo & Matriz (serie-paralelo) \\
\hline
\end{tabular}

${ }^{1}$ Efecto de las características del semiconductor.

2 Se aplicó una red neuronal MPL, sin considerar la realimentación en el modelo, lo cual representa el error reportado.

${ }^{3}$ Se puede incluir la entrada de temperatura (Tabla por horas del día), adicional a los componentes de radiación solar. Para el caso de estudio, se ha seleccionado reservar este parámetros para optimización, en el modelo el coeficiente de temperatura ha sido definido en $-0,45 \% /{ }^{\circ} \mathrm{C}$.

se convierte a par mecánico $T_{m}(t)=1 / 2 \rho \pi R$ $(t)^{3} v(t)^{2}$, dado por la ganancia del álabe captador, se obtiene el balance de torque: $J d \omega / d t=T_{m}-$ $T_{E M}-F \omega$ y la etapa de conversión de energía cinética a energía eléctrica $T_{E M}=k_{g} \varphi I$, a la salida del generador eléctrico. Se presenta la ecuación descriptiva de la etapa mecánica (5) en relación de la estructura convolucional LFSR. 


$$
T_{m}(t)=\sum_{i=1}^{n} T_{m}(i)+T_{m}(t-1)=\sum_{i=1}^{n} C_{i} \cdot v(t)+T_{m}(t-1) \therefore T_{m}(t)=C_{i} \otimes v(t)
$$

\begin{tabular}{|c|c|c|c|c|c|}
\hline \multirow{2}{*}{ Etapa } & \multirow{2}{*}{ Entrada } & \multirow{2}{*}{ Tecnología } & \multicolumn{3}{|c|}{ Correspondencia del Modelo LFSR } \\
\hline & & & Operador $(*)$ & Unidad & Sumador \\
\hline \multirow{2}{*}{ Mecánica } & \multirow{2}{*}{${ }^{1}$ Velocidad de viento $(v)$} & ${ }^{2} \mathrm{VIV}$ & Captador & Cuerpos Finitos & Eje Elástico (núcleo) \\
\hline & & Rotatoria & 3 álabes & Cámara & ${ }^{4}$ Eje Turbina \\
\hline Eléctrica & Toque $\left(T_{m}\right)$ & ${ }^{5} \mathrm{EM}$ & ${ }^{6}$ Rotor & ${ }^{7} \Delta \mathrm{V}$ & Estator \\
\hline
\end{tabular}

1 Modelo del viento. Sea $\rho$ la densidad del medio.

2 VIV: Vibraciones Inducidas por Vórtices.

${ }^{3}$ Captadores del recurso eólico.

${ }^{4}$ Modelado de estructuras por elementos finitos y osciladores acoplados, Aerodinámica de cuerpos finitos.

5 EM: Electromagnetismo.

${ }^{6}$ Secuencia Inductiva.

${ }^{7}$ Diferencia de potencial.

La correlación de la arquitectura configurable manejar las etapas del sistema de ERNC y caracterizar el sistema, los resultados se presentan en la Tabla 10, así como adaptar los aportes de los factores incidentes, a través de los coeficientes programables, para optimización.

Caso de Estudio. Modelado LFSR-ANN de Turbinas Eólicas. Turbina Eólica Comercial de las siguientes características: Diámetro $116.00 \mathrm{~m}$ y Potencia $5000 \mathrm{KW}$ (para velocidad de viento > $12 \mathrm{~m} / \mathrm{s}$ y densidad de aire referencia de $1.225 \mathrm{Kg} /$ $\mathrm{m}^{3}$ ). Datos de la ubicación seleccionada para el ensayo: Densidad de aire $(\rho) 1,21 \mathrm{Kg} / \mathrm{m}^{3}$ y rango de velocidad de viento de 2,50 a 8,60 m/s. Datos de la salida de potencia de la turbina obtenidos de la herramienta explorador de sistemas eólicos (Universidad de Chile, 2021). Los datos de entrada en entrenamiento de la MPL han sido normalizados.

Tabla 10. Modelado neuronal del arreglo eólico.

\begin{tabular}{|c|c|c|c|c|c|c|}
\hline Concentrador & \multicolumn{5}{|c|}{ Resultados del Entrenamiento de la Red Neuronal Modelado Eólico } & Realimentación \\
\hline & \multicolumn{2}{|c|}{ Etapa Mecánica } & \multicolumn{2}{|c|}{ Etapa Eléctrica } & Error 0,0108 & Capa Optimizador \\
\hline & \multicolumn{2}{|c|}{ Capa Oculta 1} & \multicolumn{2}{|c|}{ Capa Oculta 2} & Cap & Neurona Identidad \\
\hline & \multicolumn{2}{|c|}{2 N Identidad } & \multicolumn{2}{|c|}{2 N Sigmoide } & $1 \mathrm{~N} \mathrm{Id}$ & Pesos \\
\hline & Pesos & Umbral & Pesos C.0.2 & Umbral & Pesos C.S & $\mathrm{w}_{\mathrm{OPT} 1} \mathrm{w}_{\mathrm{OPT} 2}$ \\
\hline & \multirow{2}{*}{$\begin{array}{l}0,57 \\
1,86\end{array}$} & \multirow{2}{*}{$\begin{array}{r}-0,73 \\
0,83 \\
\end{array}$} & \multirow{2}{*}{$\begin{array}{c}-0,71,01 \\
-1,12-2,12\end{array}$} & \multirow{2}{*}{$\begin{array}{r}-0,71 \\
0,55 \\
\end{array}$} & $0,76-1,84$ & Umbrales Optimizador \\
\hline & & & & & Umbral 1,74 & $\mathrm{U}_{\mathrm{OPT}}$ \\
\hline & \multicolumn{2}{|c|}{$w_{O P T}$ Conf. Arreglo } & \multicolumn{2}{|c|}{$w_{O P T}$ Conf. álabes } & $w_{O P T}$ Conf. Estructural & Target: Eficiencia OPT \\
\hline Configuración óptima & \multicolumn{2}{|c|}{ Alineación: pos, h, $\alpha$} & \multicolumn{2}{|c|}{ Ángulo de pitch. $\mathrm{R}$} & Parámetros: $\mathrm{m}, \mathrm{ke}$ & MPPT, Is, Vs, Ps \\
\hline
\end{tabular}

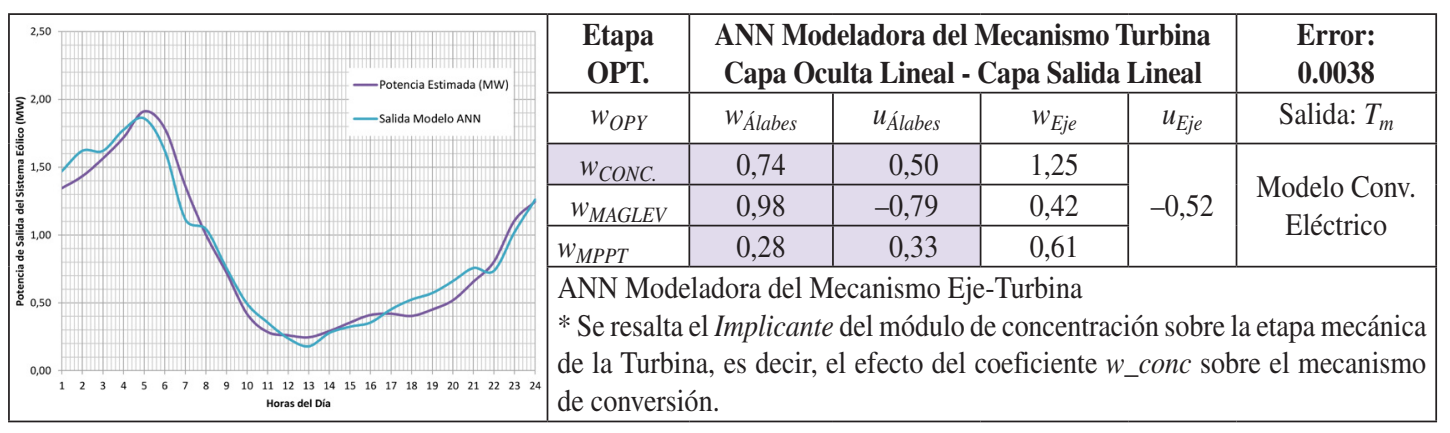


Tenemos que la selección de neuronas por número de álabes en la capa oculta permite modelar el mecanismo (eje-álabes), para la salida de Torque mecánico cálculado, con un error medio de 0.0038 converje en 100 épocas. La selección permite simplificar el procedimiento de estimación de coeficientes y optimizar la complejidad computacional. El manejo del modelo a través de dos subredes facilita el análisis, estableciendo una correspondencia entre los elementos físicos y el modelo (mapeo LFSR $\rightarrow$ ANN), con lo que se define la arquitectura de la ANN correspondiente. En la etapa mecánica se seleccionó una MPL de dos capas con 3 neuronas en la capa oculta y una neurona en la capa de salida, para la etapa eléctrica se propone una RNN con $b$ neuronas en la capa oculta, dado por el número de elementos inductores (bobinas). Esto simplifica la etapa de optimización reentrenando unicamente la subred sobre la cual se aplica el módulo de optimización.

Resulta interesante la interpretación del modelo neuronal con las variables físicas del sistema. En la etapa externa de optimización, los coeficientes tendrán como función la definición del concentrador, coeficientes de realimentación según la distribución de los aerogeneradores AG, geometría (espiral, esféricas, de efecto Venturi), cometas eólicas y manejo de válvulas de realimentación de aire comprimido, en etapas de almacenamiento CAES. Así mismo en el modelo fotovoltaico, se presenta una sola etapa de conversión o capa oculta de la red, que requirió de 3 neuronas que pueden ser asociadas a las capas del módulo fotovoltaicos. Establecido el modelo simplificado de las etapas de conversión, se establece la correlación entre los esquemas, con etapas de almacenamiento y realimentación (ver Tabla 11).

Del esquema se pueden establecer configuraciones óptimas, para las condiciones ambientales [39], en relación al control de pérdidas a través de tecnologías de refrigeración, rodamientos levitación magnética para turbinas [40], observadores de espacio de estado [41] para optimización por eficiencia de sistemas naturales, así como variables intermedias para almacenamiento directo, donde se consideran circuitos recuperadores de calor residual RCR, en líneas hidráulicas. El modelo de almacenamiento térmico de ERNC se puede relacionar con la estructura secuencial LFSR [22]. De esta manera, para la optimización se incorpora el término de realimentación, adaptando el esquema a sistemas regenerativos de alta eficiencia, así como la aplicación de algoritmos neuro-adaptativos [17, 42] para el ajuste dinámico de los coeficientes del sistema para máxima ganancia, según el esquema de optimización (Ver Tabla 12).

\section{MODELO DE OPTIMIZACIÓN DE SISTEMAS DE ERNC}

La potencia producida depende de las características del convertidor (arreglo de concentradores fotovoltaico, turbinas) por lo que los coeficientes de optimización serán determinantes a partir de la eficiencia del sistema, aplicando las técnicas de reconfiguración y realimentación selectiva. Se propone un modelo matemático generalizado en (6), basado en la estructura LFSR para diversos arreglos de ERNC y sus etapas.

$$
y(t)=\sum_{i=1}^{m} w_{i}(t) \cdot x(t)+y(t-1)
$$

La cual puede ser expresada de la forma factorial de convolución: $w_{i}(t) \otimes x(t)$ ! Donde $w_{i}(t)$ es un término que generaliza la ganancia dinámica del operador, en la posición $i$ del arreglo, sea este concentrador, convertidores de ERNC, ganancia de almacenamiento, configuración del sistema, destacando que un operador puede ser un circuito LFSR con la descripción (1), como base de concatenación en su estructura. En tanto que $x(t)$ corresponde a la señal de entrada (patrón de flujo de viento, radiación solar incidente, radiación solar concentrada, en este caso la señal es iterativa sobre el operador), la cual es un momento dado puede ser la energía convertida para almacenamiento o bien la señal reflejada entre los componentes del parque de ERNC, finalmente el parámetro $y(t-1)$ representa la realimentación inherente al sistema. Adicionalmente, la expresión puede ser controlada por selectores de realimentación y relés de selección de ramas de operadores $c_{i}$, de manera que el coeficiente de una rama define el peso correspondiente (acoplando elementos al sistema según los criterios de optimización), en el caso de ser un operador LFSR, el coeficiente ponderará la ecuación de convolución (7).

$$
y(t)=\sum_{j=1}^{n} c_{j} \cdot \alpha^{j}\left(\sum_{i=1}^{m} w_{i}(x) \cdot x(t)\right)+b_{s} \cdot \beta^{j} \cdot y(t-1)(7)
$$

La ecuación obtenida presenta auto-similitud con los diversos modelos de convertidores y sus etapas 
Tabla 11. Correlación entre esquemas de sistemas de conversión y almacenamiento de ERNC.

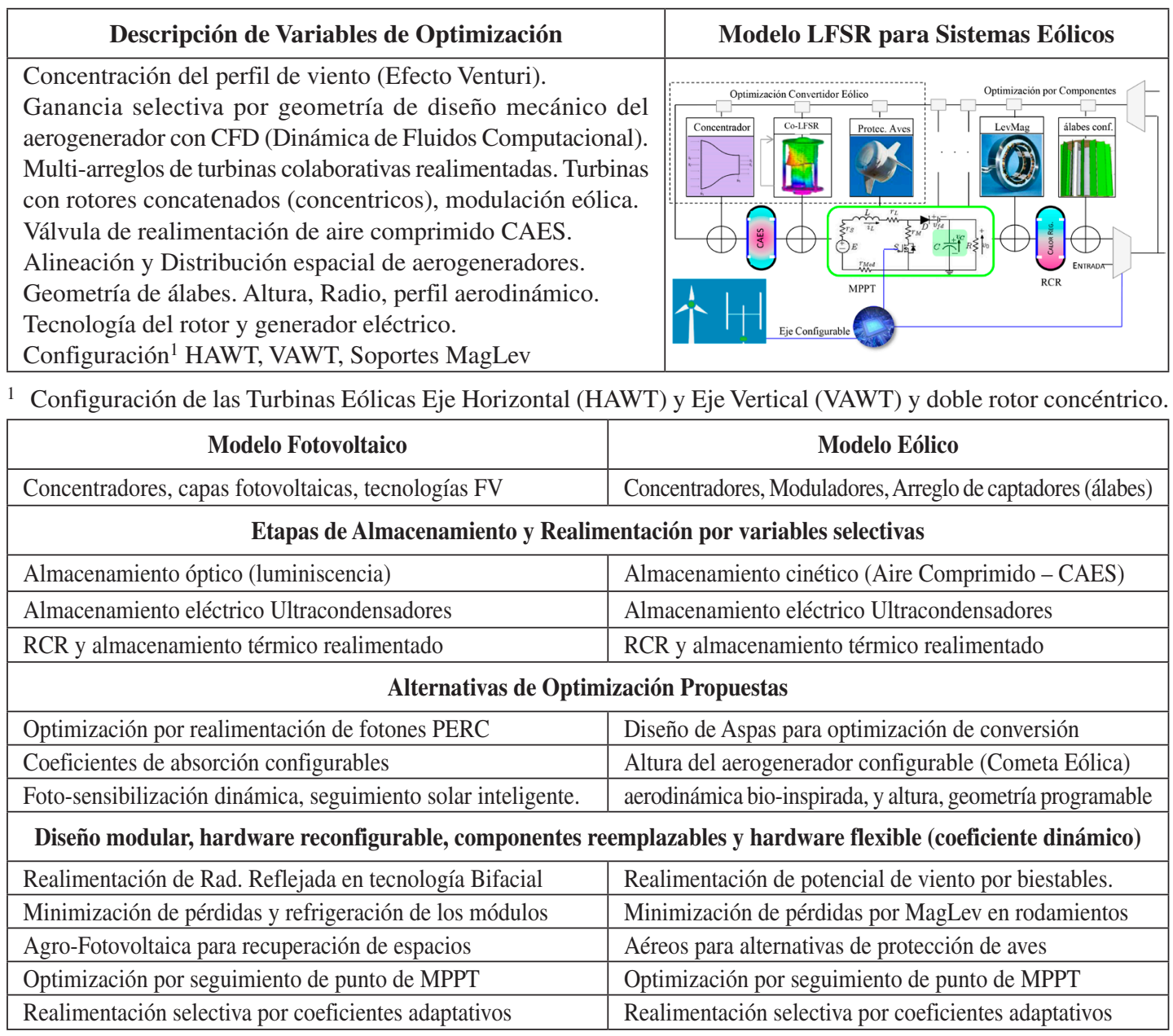

componentes, por lo que soporta diversos esquemas configurables y puede ser extrapolado, a nivel de sistemas (física clásica) e interacción de energía (física cuántica), en la aplicación de ciencia de los materiales inteligentes y resonancia, que permita postular nuevas optimizaciones $c_{j}$, en relación a los componentes de energía $\alpha^{j}, \beta^{j}$ basadas en los estados de la materia, composición reconfigurable, control magnético de parámetros y realimentación selectiva, coeficiente programable, para cada etapa y nivel fractal.

\section{Análisis de Resultados}

Luego de encontrar la correlación entre las variables del sistema físico, podemos observar que los coeficientes característicos corresponden a funciones iterativas auto-similares, que se pueden definir como funciones o coeficientes LFSR factoriales (8).

$$
\begin{gathered}
y(t)=\sum_{i=1}^{m}\left(w_{i} \cdot \theta(t)+w_{i}(t-1)\right)+w_{i}(t-1) \cdot x_{i}(t)+y(t-1) \therefore \\
y(t)=\sum_{i=1}^{m} w(t) ! \cdot x_{i}(t)+y(t-1)
\end{gathered}
$$

La optimización comprende el entrenamiento en hardware [42], control de basado en tecnología FPGA, con algoritmo adaptativo para ajustar los coeficientes al MPP y definir la combinación circuital (a través de la matriz de conmutación del arreglo) para máxima eficiencia.

Partiendo de criterios ambientales, la optimización comprende la protección de glaciares, fuentes hídricas, calidad de aire, océanos, ecosistemas, 
Tabla 12. Correspondencia de tecnologías de optimización dinámica del sistema de ERNC.

\begin{tabular}{|c|c|c|c|c|c|}
\hline \multicolumn{2}{|c|}{ Tecnología } & \multicolumn{3}{|c|}{ Descripción } & Parámetro \\
\hline \multicolumn{2}{|c|}{ Optimización Dedicada. } & \multicolumn{3}{|c|}{$\begin{array}{l}\text { Sistema de seguimiento y control de ángulo en la estructura, } \\
\text { a fin de obtener el mayor patrón incidente en su superficie. }\end{array}$} & $\begin{array}{l}\text { Ángulo de Incidencia } \\
\mathrm{I}_{\mathrm{MPPT}}, \mathrm{V}_{\mathrm{MPPT}}, \mathrm{P}_{\mathrm{MPPT}} \text {. }\end{array}$ \\
\hline \multicolumn{2}{|c|}{ Optimización Centralizada } & \multicolumn{3}{|c|}{$\begin{array}{l}\text { Una solución de direccionamiento y concentración aérea, como } \\
\text { los cometas solares propuestos en [32] y cometas eólicos. }\end{array}$} & $\begin{array}{l}\text { Set Parámetros ópticos } \\
\text { Ángulo de alineación. }\end{array}$ \\
\hline \multicolumn{2}{|c|}{ Optimización Multiplexada. } & \multicolumn{3}{|c|}{ Un mecanismo secuencial, portátil para recuperación de calor. } & Temperatura del módulo. \\
\hline \multicolumn{2}{|c|}{$\begin{array}{l}\text { Optimización por campo } \\
\text { electromagnético. }\end{array}$} & \multicolumn{3}{|c|}{$\begin{array}{l}\text { Las relaciones a nivel de unidades discretas de energía en } \\
\text { sistemas eólicos (por resonancia) y fotovoltaicos. }\end{array}$} & Parámetros de interacción de energía. \\
\hline \multirow{2}{*}{ Entrada } & \multicolumn{4}{|c|}{ Elementos del Modelo Mecánico - Etapas LFSR } & Aportaciones \\
\hline & Coef. & & escripción & Optimización configurable & Interpretación de la turbina como: \\
\hline \multirow{2}{*}{ Flujo de viento ${ }^{2}$} & $m$ & Masa de & partícula & Centro de Inercia del eje & Arreglo de captadores inteligentes. \\
\hline & $k$ & \multicolumn{2}{|c|}{ Acoplamiento } & Acoplamientos dinámicos & Registros desplazamiento con realimentación. \\
\hline Modelo/Etapas & \multicolumn{2}{|c|}{ Operador } & Registro & Sumador & Se consideran bloques discretos de energía \\
\hline Masa-Muelle & \multicolumn{2}{|c|}{$m \cdot a$} & $k \cdot x$ & $F_{G}$ & ${ }^{1}$ Se consideran los coeficientes a controlar: \\
\hline $\begin{array}{l}\text { Interpretación } \\
\text { mecánica }^{1}\end{array}$ & \multicolumn{2}{|c|}{$\begin{array}{l}\text { Transmisión de } \\
\text { E. Cinética }\end{array}$} & $\begin{array}{l}\text { Almacenamiento } \\
\text { de E. Potencial }\end{array}$ & Fuerza Resultante & $\begin{array}{l}\text { Masa: Sintonizado por campo magnético. } \\
\text { Ke: Almacenamiento de potencial elástico. }\end{array}$ \\
\hline \multicolumn{5}{|c|}{ Energía Cuántica: Spines de energía orbitando respecto a un núcleo. } & ${ }^{2}$ Optimización fractal por capas del modelo LFSR. \\
\hline
\end{tabular}

1 Se plantea un control por campo magnético para los coeficientes, a nivel de osciladores acoplados.

2 Moduladores eólicos por rotores fractales concatenados, Filtros de aire, toberas magnéticas, soportes MagLev, cometas eólicos, alineamiento inteligente (distribución fractal de álabes y turbinas), realimentación de energía residual.

flora y fauna nativa, reservas de la biosfera, así como la interacción de sus ciclos y residuos, para su reutilización circular, lo que significa la recuperación de calor, eficiencia térmica de los sistemas y control de emisiones de calor residual, siendo así la variable de optimización la realimentación de energía residual.

En los ensayos realizados se manejó el nivel de abstracción disponible, radiación solar (directa, difusa y reflejada), torque mecánico en el eje de la turbina, aplicando los cálculos para establecer las relaciones de interés. Para un control directo de cada factor se requiere de los datos intermedios, se plantea el modelo cuántico para describir el comportamiento a nivel de fotones, electrones y material de cada etapa, así como un modelo generalizado. En la Tabla 13.a se presenta el reconocimiento de patrones estructurales y Tabla 13.b el análisis de resultados.

En comparación con trabajos previos [1-2] los resultados acá presentados han logrado reconocer un modelo estructural coincidente entre la conversión fotovoltaica y eólica, detallando la descripción de la turbina rotativa mencionada en [22]. Por otra parte, el análisis de arquitectura y los modelos matemáticos del esquema configurable de la matriz energética [45] presenta una correlación con los hallazgos conceptuales e interpretación teórica de los sistemas, siendo un referente para el diagnóstico en base al modelo ANN, técnicas de análisis de fallas en turbinas eólicas [46], control adaptativo, soporte matemático, actualización y optimizaciones dinámicas.

Un aspecto novedoso consiste en la relación operadores LFC $(n, k)$ como generalizadores de sistemas de energías renovables, ahora aplicado a sistemas eólicos y componentes de conversión secuencial, estableciendo un modelo concatenado, de forma modular. Esto debido a que las redes neuronales convolucionales presentan la operación de convolución en las neuronas, con arquitectura LFSR configurable en hardware, en lugar de la multiplicación escalar. Además en base al modelo se pueden realizar estimaciones de hardware o capacidad de cómputo [47], lo que favorece los estudios de factibilidad técnicos y análisis de criterios. Finalmente, se presenta un aporte en materia ambiental para la adaptación dinámica a criterios de eficiencia energética [48], reutilización 
Tabla 13.a. Reconocimiento de patrones estructurales de sistemas de ERNC.

\begin{tabular}{|c|c|c|c|c|}
\hline Conversión/LFSR & Operador G (x) & Registro R (t) & $R(t-1)$ & Sumador \\
\hline Concentrador & Ganancia del dispositivo (Lente) & Acumulación $(\lambda)$ & FB. Reflejada & Colector de Salida \\
\hline Fotovoltaica & Ganancia del material FV & Capas del módulo, MOF & PERC, bFV & Captador e(x) \\
\hline Turbinas & Ganancia del álabe & Área de barrido del A.G. & Ciclo de giro & Eje de la Turbina \\
\hline $\begin{array}{l}\text { Convertidor } \\
\text { Mecánico-Eléctrico }\end{array}$ & $\begin{array}{l}\text { Relación entre Voltaje inducido } \\
\text { Velocidad angular de giro } \\
\text { Flujo magnético inducido }\end{array}$ & Diferencia de Potencial & Campo inducido Remanente & Fuerza Electromotriz \\
\hline \multirow{3}{*}{ Almacenamiento } & Conversión de Energía & \multirow{3}{*}{ Tanques multi-etapas } & \multirow{3}{*}{ Al sistema ERNC } & \multirow{3}{*}{ Válvulas de línea } \\
\hline & RCR (Recuperador de calor) & & & \\
\hline & Compresor de aire & & & \\
\hline
\end{tabular}

Tabla 13.b. Análisis de resultados del modelado LFSR.

\begin{tabular}{|l|l|l|}
\hline \multicolumn{1}{|c|}{ Objetivo } & \multicolumn{1}{|c|}{ Método } & \multicolumn{1}{|c|}{ Resultado } \\
\hline $\begin{array}{l}\text { Reconocer patrones en las } \\
\text { estructuras de los módulos de } \\
\text { ERNC. }\end{array}$ & $\begin{array}{l}\text { Correlación de etapas físicas y etapas de la } \\
\text { arquitectura LFSR. Modelado detallado de una } \\
\text { turbina con arquitectura LFSR. }\end{array}$ & $\begin{array}{l}\text { Descripción matemática generalizada } \\
\text { Correlación entre fotovoltaica y turbinas secuenciales. }\end{array}$ \\
\hline \multirow{2}{*}{$\begin{array}{l}\text { Modelar etapas de conversión } \\
\text { de ERNC. }\end{array}$} & $\begin{array}{l}\text { MPL: Modelo concurrente, requerimiento de } \\
\text { el cálculo de la matriz de coeficientes. }\end{array}$ & $\begin{array}{l}\text { Tratamiento de redes profundas. } \\
\text { (Interpretación de pesos, bias y F.A.). }\end{array}$ \\
\cline { 2 - 3 } & $\begin{array}{l}\text { PNN: Menor requerimiento HW, modelado } \\
\text { secuencial y multi-etapa. }\end{array}$ & $\begin{array}{l}\text { Tratamiento de redes fractales. } \\
\text { Simplificación en el método de modelado, por etapas. }\end{array}$ \\
\hline $\begin{array}{l}\text { Establecer el modelo matemático } \\
\text { para optimización, basado en } \\
\text { arquitectura LFSR y coeficientes } \\
\text { adaptativos. }\end{array}$ & $\begin{array}{l}\text { Interrelación de coeficientes. } \\
\text { Coeficientes optimizadores, en base a } \\
\text { realimentación selectiva. } \\
\text { Extrapolación del modelo. } \\
\text { Relación parámetros físicos-matemáticos. }\end{array}$ & $\begin{array}{l}\text { Modelo en función de parámetros y habilitadores } \\
\text { selectivos. } \\
\text { Operadores LFSR (configurables). } \\
\text { Versatilidad, concatenación modular. } \\
\text { Reciclaje de Tecnologías y equipos. }\end{array}$ \\
\hline $\begin{array}{l}\text { Aportes Conceptuales de la Investigación } \\
\text { Principios LFSR paralelizado [43]. Manejo de Niveles de Abstracción. Modelo LFSR generador de señales: Los componentes de } \\
\text { radiación difusa se pueden tratar alternativamente de forma secuencial, como la realimentación al sistema (señal de entrada en } t-1)\end{array}$ \\
$\begin{array}{l}\text { o bien de forma concurrente, por ser una señal estimada en relación a un modelo de radiación previo, es decir, que la operación } \\
\text { LFC, puede estar contenida en la señal o en el operador, según sea el caso. } \\
\text { La capa interna se puede manejar como una aproximación del modelo, la capa externa de optimización, aplica el modelo LFSR } \\
\text { para manejo directo de la matriz de coeficientes y asignación paramétrica, en relación a la aplicación de técnicas de optimización } \\
\text { y hardware reconfigurable IEDs [44], donde estos corresponden a los coeficientes de realimentación. }\end{array}$ \\
\hline
\end{tabular}

de infraestructura, adaptabilidad, reconfiguración dinámica y optimización en base al modelo, en el marco de desarrollo sostenible.

Una marcada diferencia con investigaciones previas, corresponde a la aplicación del operador LFSR en capas de la ANN u operadores de los coeficientes del polinomio LFSR por componente del patrón de entrada. Lo que se ha planteado corresponde a un tratamiento modular del sistema, a fin de obtener una simplificación en el método, en lo que la red supervisada emplea targets conocidos de las etapas intermedias. Es importante destacar entre los aportes del modelo LFSR que este permite la concatenación de diversas técnicas de optimización de forma colaborativa, siempre que sean compatibles (Ver Tabla 13.c), sobre los arreglos reconfigurables de turbinas (eólicas, termosolar, OTEC), convertidores resonantes (aero-elástico, hidro-elástico), fotovoltaica mPV, bPV y tecnologías de almacenamiento. La principal ventaja en la flexibilidad y modelo generalizado, que soporta la configuración modular y por ende su optimización dinámica con máxima eficacia. 
Tabla 13.c. Análisis comparativo de aportes del modelo LFSR.

\begin{tabular}{|c|c|c|c|c|}
\hline & Sistematización del Modelo & \multicolumn{3}{|c|}{ Generalización Alcanzada } \\
\hline \multirow{3}{*}{ Factor Diferenciador } & \multirow{3}{*}{$\begin{array}{l}\text { El modelo de correspondencia entre el esquema LFSR y } \\
\text { el sistema físico permite seleccionar las subredes, capas } \\
\text { y neuronas para modelado y optimización. }\end{array}$} & Turbina HAWT & MPL & Etapas: Capa Oculta \\
\hline & & Turbina VAWT & RNN & Recurrente \\
\hline & & Capa Mecánica & $\mathrm{a}$ & Nro. álabes \\
\hline \multirow{5}{*}{$\begin{array}{l}\text { Eficacia de } \\
\text { optimización, con } \\
\text { estimación de aportes: } \\
w_{11}\left[w_{A 1}(t)\right] \\
\text { en base al modelo. }\end{array}$} & \multirow{3}{*}{$\begin{array}{l}\text { La eficiencia se ve incrementada por el control de pérdidas } \\
\text { a través de la realimentación de energía residual, lo que } \\
\text { no se contempla en otros modelos. }\end{array}$} & Capa Eléctrica & $\mathrm{b}$ & Nro. bobinas \\
\hline & & Por Vibración & $\mathrm{n}$ & Nro. de Partículas \\
\hline & & Parque Eólico ${ }^{2}$ & $\mathrm{c}$ & Nro. de Turbinas \\
\hline & $\begin{array}{l}\text { La optimización matemática permite definir el objetivo, } \\
\text { por el cálculo de los máximos de eficiencia y mínimos } \\
\text { de variables ambientales, a fin de establecer las metas } \\
\text { de optimización. }\end{array}$ & \multicolumn{3}{|c|}{$\begin{array}{l}\text { Siendo c el número de turbinas o elementos } \\
\text { convertidores del arreglo colaborativo y s los } \\
\text { elementos de la capa de salida del parque. } \\
\text { ANN sobre el modelo de concatenación LFSR. }\end{array}$} \\
\hline & $\begin{array}{l}\text { Estimación de la potencia de salida objetivo. } \\
\text { Se calculan los coeficientes del modelo, matriz } w_{i, j} \\
\text { Se establecen las señales de accionamiento }\end{array}$ & \multicolumn{3}{|c|}{$\begin{array}{l}\text { Con los valores de } w_{i, j} \text { se fijan los parámetros } \\
\text { óptimos y se establecen las señales de control } \\
\text { para configuración de los convertidores. }\end{array}$} \\
\hline Aporte Incremental & $\begin{array}{l}\text { Se universaliza el modelo para diversas tecnologías de } \\
\text { ERNC. Desarrolló de modelo para turbinas generalizado } \\
\text { y modelo estructural para tecnología de conversión } \\
\text { por resonancia. Está pensado para adaptarse tanto a } \\
\text { la tecnología implementada como a NTE y avances } \\
\text { científicos, de forma progresiva. }\end{array}$ & \multicolumn{3}{|c|}{$\begin{array}{l}\text { Se puede establecer una combinación de } \\
\text { tecnologías implementadas con técnicas } \\
\text { emergentes, como }{ }^{1} \text { hologramas ópticos [49] } \\
\text { obteniendo mayor eficiencia y estimando el } \\
\text { aporte total, sobre las ecuaciones del modelo } \\
\text { desarrollado. }\end{array}$} \\
\hline \multicolumn{5}{|c|}{ Valorización de los recursos energéticos para su utilización eficiente y responsable. } \\
\hline \multicolumn{5}{|c|}{$\begin{array}{l}\text { Revalorización del potencial en infraestructura y tecnología, dentro de un programa de reutilización/reciclaje de equipos, } \\
\text { componentes, materiales y energía residual. }\end{array}$} \\
\hline \multicolumn{5}{|c|}{$\begin{array}{l}\text { Incorporación conceptual de hardware configurable como esquema de optimización del sistema, con la adaptación de la matriz } \\
\text { de coeficientes y realimentación. Componentes reemplazables, intercambiables, con modelador dinámico. }\end{array}$} \\
\hline \multicolumn{5}{|c|}{ Concatenación de Ganancias por los coeficientes de optimización sobre los coeficientes físicos o parámetros técnicos. } \\
\hline \multicolumn{5}{|c|}{ Actualización del talento humano, valorizando los conocimientos y experiencias en el sector energético [50]. } \\
\hline
\end{tabular}

${ }^{1}$ Hologramas que se pueden insertar en el panel para separar y dirigir los componentes espectrales, para aumentar la cantidad de energía convertida, aproximadamente un 5\%.

2 Define un enjambre de Turbinas Eólicas Reconfigurables - TER inteligentes.

Una ventaja del modelo es su compatibilidad para abarcar una amplia gama de aplicaciones en el área de energía renovables por la correspondencia estructural y matemática para las configuraciones de los convertidores de ERNC, así como el soporte de métodos de optimización matemático basado en el modelo, para el sistema colaborativo [51], universalizando el modelo a fin de habilitar etapas de la estructura, de forma selectiva. En el caso de las HAWT se puede omitir el registro de inercia del eje para simplificar el esquema a un arreglo paralelo de elementos de captación eólica, el cual a través de un algoritmo adaptativo permite fijar los valores óptimos de los coeficientes convirtiéndolo en un arreglo inteligente. Además se han estudiado los niveles de abstracción, interpretando las estructuras de osciladores acoplados [52], lo que permite tener control y sintonización de coeficientes LFSR.
El modelo sistematizado soporta la estimación del aporte de cada técnica empírica y el análisis por medio de métodos de optimización matemático [47]. La arquitectura del modelo con esquema LFSR permite generar secuencias fractales, el mismo presenta auto-similitud para diversas etapas de los mecanismos, a nivel de mecánica cuántica, física moderna y ondas [52], lo que establece patrones con relación $\phi$, estos patrones de orden fractal resultan de interés para la geometría de los álabes [53], alineación y disposición de los elementos en el tejido del parque eólico, con el objetivo de maximizar el área de captación efectiva, siendo este un nuevo coeficiente de optimización. antes no considerado para aprovechamiento de la fuente de ERNC.

El modelo LFSR desarrollado permite optimizar la ganancia bifacial en un rango dado [11-12] y 
la combinación de técnicas de optimización. Así como la eficiencia de arreglos de turbinas eólicas, considerando los resultados reportados en [49], como efecto de la interacción sobre las turbinas aguas abajo, la configuración y alineación entre elementos del parque eólico. Esto considerando el efecto de modulación de patrón de energía incidente, por patrón reflejado entre elementos anidados.

\section{CONCLUSIONES}

Gracias al modelo desarrollado, se obtiene un método de optimización para esquemas de ERNC híbridos, a través de coeficientes adaptativos. Con esto se pueden lograr resultados más eficientes, dentro de un esquema reconfigurable, con alto grado de flexibilidad en hardware, para la digitalización de la infraestructura y configuración de forma remota de etapas y componentes. La optimización basada en el modelo matemático permite aplicar técnicas avanzadas: Planificación de ganancia por capas, control de esquemas variables en reconfiguración dinámica del modelo y hardware en lazo, usando tecnología FPGA.

De esta manera, partiendo del compromiso de mitigar los efectos de contaminación térmica, para la conservación del hábitat de la fauna y mínimo impacto ambiental, se planteó la optimización por realimentación, minimización de pérdidas (líneas de transporte con material termoaislante), RCR y captadores térmicos, soportes de levitación magnética, para mejorar la eficiencia y aprovechamiento del gradiente térmico y energía residual, en circuitos cerrados, disminuyendo el impacto ambiental por disipación de calor, incluidas aplicaciones de fotovoltaica bifacial y cogeneración térmica.

Incorpora nuevos conceptos tal como unidades discretas de energía eólica, modulación de patrón de flujo incidente, coeficientes fractales polinómicos. Por lo que en los resultados del modelo aparecen términos, dando una gama de coeficientes adaptativos para la optimización y un tratamiento del parque de ERNC. El manejo etapas aporta modularidad, escalabilidad y capacidad de reconfiguración dinámica del arreglo inteligente, almacenamiento, realimentación selectiva, con componentes reemplazables y reutilizables dentro del concepto de hardware circular, a fin de facilitar la actualización y optimización dinámica, sobre un modelo LFSR compatible para las nuevas tecnologías y desarrollo sostenible.

Otro aspecto mencionado en la investigación corresponde al método que se aplica en el modelado neuronal depende del nivel de abstracción, en relación con el conjunto de datos disponibles, lo que permite identificar una matriz caracterizadora de las propiedades físicas, al aplicar redes generalizadoras de múltiples capas, la arquitectura de la ANN permite hacer coincidir los coeficientes del modelo LFSR, con los pesos sinápticos. Lo importante a resaltar es que el modelo simplifica el manejo de las variables de comportamiento y operadores, para establecer los coeficientes de optimización, que inciden específicamente sobre la variable a optimizar, identificando una estructura fractal.

Se obtiene finalmente, un modelo que incorpora el componente de interacción de energía, de manera formal en el modelo matemático, permitiendo sustentar un modelador-optimizador, a través de los parámetros con impacto en el rendimiento del sistema. Tal es el caso, de la realimentación directa de ERNC en el sistema almacenamiento, para mejorar la eficiencia, aplicando concentradores solares ópticos, refrigeración forzada para control del índice de rendimiento térmico, posicionamiento del arreglo, MPPT coordinado con el circuito de electrónica de potencia.

\section{REFERENCIAS}

[1] C. Sandoval-Ruiz. "Arreglo Inteligente de Concentración Solar FV para MPPT usando Tecnología FPGA". Revista Técnica Facultad de Ingeniería Universidad del Zulia. Vol. 43 $\mathrm{N}^{\circ}$ 3, pp. 122-133, 2020. ISSN: 0254-0770. DOI: $10.22209 /$ rt.v43n3a02.

[2] C. Sandoval-Ruiz. "Arreglos Fotovoltaicos Inteligentes con Modelo LFSR-Reconfigurable". Ingeniería. Revista de la Universidad de Costa Rica. Vol. 30 N $^{\circ}$ 2, pp. 32-61. 2020. ISSN: 2215-2652. DOI: 10.15517/RI.V30I2.39484.

[3] C. Sandoval-Ruiz. "Tecnología R-IEDs para ERNC, Teletrabajo y Mitigación de Impacto Ambiental". Industrial Data. Vol. $23 \mathrm{~N}^{\circ} 2$, pp. 151-167. 2020. ISSN: 1810-9993. DOI: 10.15381/idata.v23i2.18633. 
[4] M. Hossain and H. Mahmood. "Short-Term Photovoltaic Power Forecasting Using an LSTM Neural Network and Synthetic Weather Forecast". IEEE Access. Vol. 8, pp. 172524-172533. 2020. ISSN: 2169-3536. DOI: 10.1109/ACCESS.2020.3024901.

[5] S. Al-Dahidi, M. Louzazni and N. Omran. "A local training strategy-based artificial neural network for predicting the power production of solar photovoltaic systems". IEEE Access. Vol. 8. pp. 150262-150281. 2020. ISSN: 2169-3536. DOI: 10.1109/ ACCESS.2020.3016165.

[6] K. Chiteka, R. Arora and S. Sridhara. "A method to predict solar photovoltaic soiling using artificial neural networks and multiple linear regression models". Energy Systems. Vol. 11 No 4, pp. 981-1002. 2020. ISSN: 18683967. DOI: 10.1007/ s12667-019-00348-w.

[7] K. Garud, S. Jayaraj and M. Lee. "A review on modeling of solar photovoltaic systems using artificial neural networks, fuzzy logic, genetic algorithm and hybrid models". Intern. Journal of Energy Research. Vol. 45, pp. 6-35. 2021. ISSN: 0363-907X. DOI: 10.1002/ er.5608.

[8] C. Herrera. "Evaluación de técnicas de aprendizaje profundo para el pronóstico de generación eléctrica en corto plazo de un sistema fotovoltaico de $16 \mathrm{~kW}$ ". Tesis Universidad de Chile, 2020.

[9] A. Al-Waeli, H. Kazem, J. Yousif, M. Chaichan and K. Sopian. "Mathematical and neural network modeling for predicting and analyzing of nanofluid-nano PCM photovoltaic thermal systems performance". Renewable Energy. Vol. 145, pp. 963-980. 2020. ISSN: 1879-0682. DOI: 10.1016/j. renene. 2019.06.099.

[10] S. Gowid and A. Massoud. "A robust experimental-based artificial neural network approach for photovoltaic maximum power point identification considering electrical, thermal and meteorological impact". Alexandria Engineering Journal. Vol. 59 No 5, pp. 3699-3707. 2020. ISSN: 11100168. DOI: $10.1016 /$ j.aej.2020.06.024.

[11] W. Gu, et al. "A comprehensive review and outlook of bifacial photovoltaic (bPV) technology". Energy Conversion and Management. Vol. 223, pp. 113283. 2020. ISSN: 0196-8904. DOI: 10.1016/j. enconman.2020.113283.

[12] W. Gu, et al. "A coupled optical-electricalthermal model of the bifacial photovoltaic module". Applied Energy. Vol. 258, pp. 114075. 2020. ISSN: 1872-9118. DOI: 10.1016/j.apenergy.2019.114075.

[13] J. González. "Utilización de ultracondensadores para la mejora de eficiencia de una instalación fotovoltaica". Tesis Doctoral. Universidad de Valladolid. 2015.

[14] D. González-Montoya, et al. "Reconfiguración de paneles fotovoltaicos para reducción del consumo de hidrógeno en las celdas de combustible de sistemas híbridos". Tecnológicas. Vol. $20 \mathrm{~N}^{\mathrm{o}}$ 39, pp. 85-99. 2017. ISSN: 0123-7799.

[15] S. Cabello-García. "Reconfiguración dinámica del campo solar de una planta fotovoltaica para maximizar la producción de energía". Tesis Universidad de Sevilla. Sevilla, España. 2018.

[16] C. Sandoval-Ruiz. "Operador matemático LFC (n, k) en campos finitos basado en concatenación fractal para $\mathrm{GF}(2 \mathrm{~m})$ Extendido". Ciencia e Ingeniería. Vol. 41 $\mathrm{N}^{\circ}$ 2, pp. 197-204. 2020. ISSN: 2244-8780.

[17] C. Sandoval-Ruiz. "LFSR-Fractal ANN Model applied in R-IEDs for Smart Energy". IEEE Latin America Transactions. Vol. 18 No 4, pp. 677-686. 2020. DOI: 10.1109/ TLA. 2020.9082210.

[18] C. Sandoval-Ruiz, "Fractal Mathematical over Extended Finite Fields Fp[x]/(f(x))". Proyecciones Journal of Mathematics. Vol. 40 $\mathrm{N}^{\circ} 3$, pp. 731-742. 2021. ISSN: 0717-6279. DOI: 10.22199/issn.0717-6279-4322.

[19] F. Reyniel. "Sistemas fotovoltaicos con módulos bifaciales: estudio comparativo de su rendimiento (PR) en distintas condiciones de irradiancia y temperatura". Tesis de Maestría. Universidad de Cartagena. España. 2019.

[20] J. Guamán, et al. "Análisis comparativo de los modelos dinámicos de una turbina eólica de velocidad fija". Ingenius. $\mathrm{N}^{\mathrm{o}} 15$, pp. 37-47. 2016. ISSN: 1390-650X. DOI: 10.17163/ings.n15.2016.04.

[21] S. Brusca, G. Capizzi, G. Lo Sciuto and G. Susi. "A new design methodology to predict wind farm energy production by means of 
a spiking neural network-based system". International Journal of Numerical Modelling: Electronic Networks, Devices and Fields. Vol. $32 \mathrm{~N}^{\circ}$ 4. DOI: 10.1002/jnm.2267. 2019.

[22] C. Sandoval-Ruiz. "Plataforma de Investigación de Redes Eléctricas Reconfigurables de Energías Renovables aplicando Modelos LFSR". UCT. Vol. 23 No 95, pp. 103-115. 2019. ISSN 1316-4821.

[23] C. Sandoval-Ruiz. "Modelo VHDL de Control Neuronal sobre tecnología FPGA orientado a Aplicaciones Sostenibles". Ingeniare. Vol. 27 $\mathrm{N}^{\mathrm{o}}$ 3, pp. 383-395. 2019. ISSN: 0718-3305. DOI: $10.4067 /$ S0718-33052019000300383.

[24] P. Herrán-Funes. "Diseño de implantación y análisis del potencial de generación de sistemas fotovoltaicos flotantes en centrales hidroeléctricas reversibles en España”. Madrid,España. 2018.

[25] S. Medel-Caro. "Estudio de implantación de tecnologías mareomotrices y undimotrices como pequeños medios de generación distribuida". Tesis Universidad de Chile. Santiago, Chile. 2010.

[26] R. Castillo, V. Segovia, G. Díaz and C. Araya. "Potencial undimotriz de la región de Tarapacá (Chile) como foco de fomento productivo". Ingeniare. Vol. $26 \mathrm{~N}^{\circ}$ 1, pp. 72-85. 2018. DOI: 0.4067/S0718-33052018000100072.

[27] P. Bilbao-Rojas. "Dimensionamiento óptimo de sistemas de almacenamiento para centrales eólicas". Tesis Universidad de Chile. Santiago. Chile. 2013.

[28] A. Reinoso. "Dimensionamiento óptimo y análisis técnico económico de un Sistema de Almacenamiento de Energía". Tesis Universidad de Chile. Chile. 2018.

[29] K. Griffiths, N. Halcovitch, and J. Griffin. "Long-Term Solar Energy Storage under Ambient Conditions in a MOF-Based SolidSolid Phase-Change Material". Chemistry of Materials. 2020. DOI: 10.1021/acs. chemmater.0c02708.

[30] C. Sandoval-Ruiz. "Proyecto Cometa Solar - CS para optimización de sistemas fotovoltaicos". UCT. Vol. 24 N $^{\circ}$ 99, pp. 74-87. 2020. ISSN 1316-4821.

[31] M. Brazo-Reina. "Sistema pasivo de almacenamiento de energía térmica en centrales termosolares". Tesis Universidad de Sevilla. Sevilla, España. 2016.
[32] M. Monar-González. "Sistema de almacenamiento de energía térmica basado en vapor en centrales termosolares. Análisis y modelado". Tesis Universidad de Sevilla. Sevilla, España. 2017.

[33] D. Chlipf, R. Stepper and G. Schneider. "Tecnología de Almacenamiento de CSP". 2014.

[34] CEMIE-Océano. "Conversión de Energía Térmica Oceánica (OTEC)". Colección Energías Renovables del Océano. México. 2017.

[35] GIZ. "Plantean reconversión de termoeléctricas a carbón gracias energías renovables". 2019. Fecha de Consulta: 12 de Enero de 2021. URL: 4echile.cl/ plantean-reconversion-termoelectricascarbon-gracias-energias-renovables/

[36] SEA. "Guía para la Descripción de Proyectos de Centrales Solares de G.E.E en el SEIA". 1ra Edición. Servicio de Evaluación Ambiental. Chile. 2017.

[37] SEA. "Guía para la Evaluación de Impacto Ambiental de Centrales Eólicas de G.E.E en el SEIA". 2da Edición. Servicio de Evaluación Ambiental. Chile. 2020.

[38] Universidad de Chile. "Explorador Solar". 2021. Fecha de consulta: 20 de Mayo de 2020. URL: http://ernc.dgf.uchile.cl:48080/ inicio

[39] A. Zurita, et al. "State of the art and future prospects for solar PV development in Chile". Renewable and Sustainable Energy Reviews. Vol. 92, pp. 701-727. 2018.

[40] Almazan-Arvizu, R. et.al. "Control PI difuso de un sistema de levitación magnética mediante un sistema embebido". Ingeniería, investigación y tecnología. Vol. $20 \mathrm{~N}^{\circ} 4$. 2019.

[41] Zamarreño, J. and Merino, A. "Red neuronal estructurada en el espacio de estados como modelo de caja gris". In XL Jornadas de Automática, pp. 639-646. Coruña, España. 2019.

[42] C. Sandoval-Ruíz. "Modelo Neuro-Adaptativo en VHDL, basado en Circuitos NLFSR, para Control de un Sistema Inteligente de Tecnología Sostenible". UCT. Vol. 21 N 85, pp. 140-149. 2017. ISSN 1316-4821.

[43] C. Sandoval-Ruiz. "Modelo Optimizado del Codificador Reed-Solomon $(255, \mathrm{k})$ en 
VHDL a través de un LFSR paralelizado". Tesis Doctoral Universidad de Carabobo. Venezuela. 2013.

[44] C. Sandoval-Ruiz. "Códigos Reed Solomon para Sistemas Distribuidos de Energías Renovables y Smart Grids a través de Dispositivos Electrónicos Inteligentes sobre Tecnología FPGA”. Memorias. Investigación en Ingeniería. Vol. $16 \mathrm{~N}^{\circ}$ 1, pp. 37-54. 2018. ISSN: 2301-1106.

[45] C. Sandoval-Ruiz. "Laboratorio móvil para optimización de sistemas de energías renovables y aplicaciones ambientales". Ciencia e Ing. Vol. $42 \mathrm{~N}^{\mathrm{o}}$ 2, pp. 169-178. 2021. ISSN: 1316-7081

[46] M. Mago and E. Bulla. "Métodos no Convencionales para evaluar fallas en transformadores de distribución". IPAPEDIEdiciones Signo. 1ra Edición, pp. 27-29. Valencia, Venezuela. ISBN: 978-980-181722-2. 2021.

[47] C. Sandoval-Ruiz. "Métodos Numéricos en Diferencias Finitas para la Estimación de Recursos de Hardware FPGA en arquitecturas LFSR $(n, k)$ Fractales". Revista IIT. Vol. 20 $\mathrm{N}^{\circ} 3$, pp. 1-10. 2019. ISSN: 2594-0732. DOI: 10.22201/fi.25940732e.2019.20n3.032.

[48] C. Sandoval-Ruiz. "Smart systems for the protection of ecosystems, flora and fauna".
UCT. Vol. $25 \mathrm{~N}^{\circ} 110$, pp. 138-154, 2021. ISSN 1316-4821. DOI: 10.47460/uct.v25i110.486.

[49] J. Zhao, P. Chrysler and R. Kostuk. "Holographic low concentration optical system increasing light collection efficiency of regular solar panels". Journal of Photonics for Energy. Vol. $11 \mathrm{~N}^{\circ}$ 2, pp. 027002. 2021. ISSN: 1947-7988. DOI: 10.1117/1. JPE.11.027002.

[50] C. Sandoval-Ruiz. "Capacitación remota en competencias técnicas en el marco de la ambientalización de la Ingeniería". Eduweb. Vol. $15 \mathrm{~N}^{\mathrm{o}}$ 2, pp. 10-21. 2021. ISSN: 1856-7576. DOI: 10.46502/ issn.1856-7576/2021.15.02.1.

[51] J. Leithon, et al. "Energy optimization through cooperative storage management: A calculus of variations approach". Renewable Energy. Vol. 171, pp. 1357-1370. 2021. ISSN: 18790682. DOI: 10.1016/j.renene.2021.02.093

[52] A. French. "Vibraciones y Ondas". 1ra Edición. Editorial Reverté. pp. 89-182. Barcelona, España. ISBN: 84-291-4100-6. 1974.

[53] Y. Patil. "Design, Fabrication and Analysis of Fibonacci Spiral Horizontal Axis Wind Turbine". International Journal of Aerospace and Mechanical Engineering. Vol. $5 \mathrm{~N}^{\mathrm{o}} 1$. 2018. ISSN: 2393-8609. 